\title{
Chaotic Mixing of Tracer and Vorticity by Modulated Traveling Rossby Waves
}

R. T. Pierrehumbert

Department of Geophysical Sciences

University of Chicago

Chicago, IL 


\begin{abstract}
We consider the mixing of passive tracers and vorticity by temporally fluctuating large scale flows in two dimensions. In analyzing this problem, we employ modern developments stemming from properties of Hamiltonian chaos in the particle trajectories; these developments generally come under the heading "chaotic advection" or "lagrangian turbulence." A review of the salient properties of this kind of mixing, and the mathematics used to analyze it, is presented in the context of passive tracer mixing by a vacillating barotropic Rossby wave. We then take up the characterization of subtler aspects of the mixing. It is shown the chaotic advection produces very nonlocal mixing which cannot be represented by eddy diffusivity. Also, the power spectrum of the tracer field is found to be $\mathrm{k}^{-1}$ at shortwaves - precisely as for mixing by homogeneous, isotropic two dimensional turbulence, - even though the physics of the present case is very different. We have produced two independent arguments accounting for this behavior.

We then examine integrations of the unforced barotropic vorticity equation with initial conditions chosen to give a large scale streamline geometry similar to that analyzed in the passive case. It is found that vorticity mixing
\end{abstract}

proceeds along lines similar to passive tracer mixing. Broad regions of homogenized vorticity ultimately surround the separatrices of the large scale streamline pattern, with vorticity gradients limited to nonchaotic regions (regions of tori) in the corresponding passive problem.

Vorticity in the chaotic zone takes the form of an arrangement of strands which become progressively finer in scale and progressively more densely packed; this process transfers enstrophy to small scales. Although the enstrophy cascade is entirely controlled by the large scale wave, the shortwave enstrophy spectrum ultimately takes on the classical $\mathrm{k}^{-1}$ form. If one accepts that the enstrophy cascade is indeed mediated by chaotic advection, this is the expected behavior. The extreme form of nonlocality (in wavenumber space) manifest in this example casts some doubt on the traditional picture of enstrophy cascade in the Atmosphere, which is based on homogeneous $2 \mathrm{D}$ turbulence theory. We advance the conjecture that these transfers are in large measure attributable to large scale, low frequency, planetary waves.

Upscale energy transfers amplifying the large scale wave do indeed occur in the course of the above-described process. However, the energy transfer is complete long before vorticity mixing has gotten very far, and therefore has 
little to do with chaotic advection. In this sense, the vorticity involved in the enstrophy cascade is "fossil vorticity," which has already given up its energy to the large scale.

We conclude with some speculations concerning statistical mechanics of two dimensional flow, prompted by our finding that flows with identical initial energy and enstrophy can culminate in very different final states. We also outline prospects for further applications of chaotic mixing in atmospheric problems. 


\section{Introduction}

Hoskins, et al. (1985) have made an eloquent case for the analysis of atmospheric and oceanic dynamics in terms of potential vorticity evolution on isentropic surfaces. These analyses reveal the hidden complexity that cannot be detected in streamfunction or geopotential height fields. At the elemental level, the smoothness of the streamfunction is simply a consequence of its being related to potential vorticity by an inverse laplacian operator. This remark does not go far enough, though, as it is the streamfunction that governs the vorticity advection; hence its smoothness has important dynamical consequences. Indeed, linear wave theory (which generally involves smooth fields) has proved a very productive approach to a range of atmospheric phenomena, and it is not at first glance easy to reconcile this evident success with the spatial complexity of observed potential vorticity fields.

Recent advances in the area known as "chaotic advection" or "lagrangian turbulence" provides a way of putting the wave/turbulence jigsaw puzzle back together. This work has demonstrated that organized large scale (even deterministic) flow patterns can produce spatially complex tracer patterns which lead to effective mixing; it also provides a powerful apparatus for characterizing the mixing (Aref 1984, Chien et al. 1986,Khakar et al. 1986, Ottino et al., 1988, and Wiggins 1988, Ottino 1989, Rom-Kedar et al 1990, inter alia). The theory predicts enhanced mixing whenever a streamfunction exhibiting a closed streamline region bounded by a separatrix (a streamline terminating in stagnation points) is subjected to temporal fluctuations. This geometry occurs whenever there is an isolated eddy embedded in a background current, and is ubiquitous in large scale atmospheric and oceanic flows. Most of the progress has been for passive tracer advection in timeperiodic flows, and it is not a priori clear whether any of these results carry over to mixing of active tracers (like potential vorticity or potential temperature) in more complex flows. Exploring this connection is the central concern of this paper.

We approach this problem by analyzing passive tracer mixing by vacillating barotropic Rossby waves, and then examining integrations of the full barotropic vorticity equation for the characteristic signature of chaotic mixing in the vorticity fields. This example illustrates the peaceful coexistence between an organized large scale wave and complex vorticity evolution leading to an enstrophy cascade. Along the way we derive some novel results on the passive 
tracer problem, particularly concerning use of two-particle correlation functions to characterize the mixing, and the relation of these functions to power spectra of the tracer. These results have clear implications for the representation of mixing in chemical tracer problems, such as ozone evolution. Our main concern, though, is with the consequences for potential vorticity mixing.

The key result is that chaotic advection theory provides considerable insight as to where potential vorticity will homogenize, and where strong gradients can survive. Potential vorticity homogenization is central to the understanding of a variety of natural phenomena, including the stratospheric polar vortex(McIntyre \& Palmer 1983), Rossby wave critical level reflection (Killworth \& McIntyre 1985), thermocline theory (Rhines and Young 1982), and Jupiter's Great Red Spot, where homogenization is commonly invoked to suppress radiation of Rossby waves (Marcus 1988). Our results demonstrate that eddy viscosity need not be stipulated to account for homogenization.

The Rossby wave problem also leads us naturally into the consideration of the properties of two-dimensional turbulence in the presence of large scale inhomogeneity. Most 2D turbulence studies, both theoretical and numerical, have been carried out under circumstances favoring homogeneity and isotropy. Certainly, this problem has a great deal of intrinsic intellectual appeal, but lurking in the background of much of this work is the hope that universal homogeneous behavior applies at scales far removed from that of the large scale inhomogeneity. Because of the nonlocality in wavenumber space of the enstrophy cascading subrange (Kraichnan, 1971), this is a dubious proposition. We shall see that an energetic large scale wave controls the enstrophy cascade down to arbitrarily small scales. However, owing to certain universal properties of chaotic advection, a classical $\mathrm{k}^{-1}$ enstrophy spectrum nevertheless emerges. Given the prominence of planetary waves in the Earth's atmosphere, it is likely that the chaotic advection model provides a better metaphor for the enstrophy cascade than isotropic, homogeneous 2D turbulence.

We also discuss the energy transfers associated with the distortion of small scale eddies by the planetary wave. The large scale wave effectively scavenges the energy of the small eddies, but it will be seen that these transfers occur at a very early stage of the game. The eddies give up their energy to the large scale long before the vorticity mixing has gotten underway. Hence the chaotic mixing properties are of little relevance to the energy transfer. 
The mathematical statement of the problem of passive tracer advection by a superposition of Rossby waves is given in $\$ 2$, along with a review of its basic behavior. Some new quantitative characterizations of the mixing, and their connection with power spectra of the concentration distribution, are given in $\S 3$. Fully nonlinear simulations of the barotropic vorticity equations establishing a connection of these results with potential vorticity mixing are given in $\S 4$. In $\S 5$ we discuss the upscale energy transfers associated with mixing of small scale vorticity. Our principal conclusions are discussed in $\S 6$, where we also set out some guideposts for future geophysical applications of chaotic mixing.

\section{Passive tracer advection: the basic behavior}

The whole chaotic advection enterprise stems from the following simple observation. If $\psi(\mathrm{x}, \mathrm{y}, \mathrm{t})$ is the streamfunction for a time-dependent incompressible flow in two dimensions, then the equations for the position $(\mathrm{X}, \mathrm{Y})$ of a marker particle are

$$
\frac{\mathrm{dX}}{\mathrm{dt}}=-\partial_{\mathrm{y}} \psi(\mathrm{X}, \mathrm{Y}, \mathrm{t}) \quad \frac{\mathrm{dY}}{\mathrm{dt}}=\partial_{\mathrm{X}} \psi(\mathrm{X}, \mathrm{Y}, \mathrm{t})
$$

Since $\psi$ is a nonlinear function of $\mathrm{X}$ and $\mathrm{Y}$, this is a nonlinear system. Moreover, if the time dependence is periodic, then this is a three degree of freedom nonlinear system, since one must specify $X, Y$ and the phase of the oscillation to determine the future course of the system. This is the minimum number of degrees of freedom necessary to support chaos. Hence the trajectories of a passive tracer particle can be chaotic even in spatially simple flow fields; this has profound implications for mixing. A further attractive feature of (1) is that it is a Hamiltonian system, with canonical coordinates $\mathrm{X}$ and $\mathrm{Y}$ and Hamiltonian $\psi$. Hence the full arsenal of techniques for dealing with Hamiltonian chaos developed over a century or more of research is available for one's employment in the investigation of such systems. The work of Aref (1984) drew attention to this opportunity, and in conjunction with the appearance of the textbook by Holmes and Guckenheimer (1983) has certainly stimulated much of the current progress in the subject. Certain antecedents concerning mixing by spatially organized flows can also be found in the much earlier paper by Welander (1955), which was certainly ahead of its time, being among the first papers to employ fractal geometry in the investigation of fluid phenomena.

In this paper, we will be concerned with particle advection by the velocity field of a superposition of two 
Rossby waves on the barotropic $\beta$-plane. The streamfunction is

$\psi=\mathrm{A} \sin \left(\mathrm{k}_{1}\left(\mathrm{x}-\mathrm{c}_{1} \mathrm{t}\right)\right) \sin \left(\mathrm{l}_{1} \mathrm{y}\right)+\varepsilon \sin \left(\mathrm{k}_{2}\left(\mathrm{x}-\mathrm{c}_{2} \mathrm{t}\right)\right) \sin \left(\mathrm{l}_{2} \mathrm{y}\right)(2)$ where the phase speeds are $c_{j}=-\beta /\left(k_{j}^{2}+l_{j}^{2}\right)$. We adopt units such that $l_{1}=1$ and $\beta=1$; further, in all results presented below $\mathrm{k}_{1}=1$ and $\mathrm{k}_{2}$ and $\mathrm{l}_{2}$ are integers, whence the flow is confined to a channel periodic in $\mathrm{x}$ with period $2 \pi$ and bounded by walls at $y=0$ and $\pi$. When $\varepsilon=0$, (1) is an exact solution of the barotropic vorticity equation for arbitrary A. The form of the perturbation is correct only in the limit of small $A$ and $\varepsilon$; however, experiments with various $\left(\mathrm{k}_{2}, \mathrm{l}_{2}\right)$ (to be discussed later) suggest that the results are not very sensitive to the spatial structure of the perturbation, being controlled primarily by the streamline geometry of the unperturbed flow and the velocity amplitude of the perturbation. Hence we are confident that the results we present for finite A will survive a more accurate specification of the velocity field.

In the comoving reference frame $x^{\prime}=x-c_{1} t$, describes a steady velocity field perturbed by a field oscillating in time with period $\mathrm{T}=2 \pi /\left(\mathrm{k}_{2}\left(\mathrm{c}_{1}-\mathrm{c}_{2}\right)\right)$, and constitutes a perturbed planar Hamiltonian system. Figure 1 shows the unperturbed streamlines in the comoving frame for $A=1$. This is the value of $A$ used throughout this paper, unless otherwise noted. There are two stagnation points (marked $\mathrm{P}$ and $\mathrm{P}^{\prime}$ ) on the lower boundary, where a particle will remain forever if so placed initially. They are both saddle points in the sense that the linearized behavior in the neighborhood of these points exhibits one stable and one unstable direction. The stagnation points $\mathrm{P}$ and $\mathrm{P}^{\prime}$ form what is known as a heteroclinic cycle, being connected by the upper arc $\mathrm{PP}^{\prime}$ (the unstable manifold of $\mathrm{P}$, which is exactly coincident with the stable manifold of $\mathrm{P}^{\prime}$ ) and the lower segment $\mathrm{P}^{\prime} \mathrm{P}$ (the unstable manifold of $\mathrm{P}^{\prime}$ which is exactly coincident with the stable manifold of $\mathrm{P}$ ). Because of the boundary conditions, the latter is preserved under perturbation; however, as $\varepsilon$ is increased from zero the former generically breaks up into a chaotic set. There is a deep and extensive literature on the association between heteroclinic (or homoclinic) cycles and chaos in perturbed Hamiltonian systems. An introduction to the subject can be found in Guckenheimer \& Holmes (1983).

The unperturbed wave has precisely the same streamline geometry as that analyzed by Knobloch \& Weiss (1987) and Weiss and Knobloch (1989), though the class of perturbations we consider is somewhat broader. These authors were motivated by modulated traveling waves appearing in thermosolutal convection, but clearly 
appreciated that their results were applicable to modulated traveling waves generally. The Rossby wave problem we consider here has the particular attraction that the basic state is an exact nonlinear solution of the inviscid equations for arbitrary amplitude; it also lends itself naturally to nonlinear simulations probing the connection between passive tracer and vorticity mixing.

The results presented in this Section should for the most part be regarded as a review of the salient properties of the system as established by Weiss \& Knobloch, though we discuss the behavior from a somewhat different perspective, and in a few instances extend the results in a modest way. An important departure from the system considered by Weiss and Knobloch is that we impose a reentrant condition on the tracer, so that material transported out of the right of the domain is reintroduced at the left; Knobloch and Weiss were principally concerned with transport amongst gyres in an infinitely long wavetrain, and so did not impose a singleperiod reentrant condition.

The Poincare map $\mathbf{P}$ provides a convenient means of analyzing the structure of the particle trajectories, when the advecting flow is time-periodic. This map takes the position $(\mathrm{x}, \mathrm{y})$ of a particle on the plane to its position one oscillation period $\mathrm{T}$ later. The sequence of points $\left(\mathrm{x}_{\mathrm{i}}, \mathrm{y}_{\mathrm{i}}\right)$ obtained by iterating the map on some initial condition is known as an orbit of the Poincare map. From an experimental standpoint, plotting an orbit amounts to taking a stroboscopic picture of the position of a single marker particle every time the advecting flow swings around to the same state.

Figure 2 shows a family of numerically computed Poincare orbits starting from a number of different initial conditions for $\varepsilon=.1$ and $\left(\mathrm{k}_{2}, \mathrm{l}_{2}\right)=(1,2)$. The blue and green orbits trace out a series of disjoint, smooth curves. Orbits of this type are known as tori. The terminology is not obscure if one recalls that the Poincare sections are a $2 \mathrm{D}$ cut of a 3D object (think of the intersection of a plane with an inflated inner-tube). Orbits in the tori are nonchaotic; they are characterized by zero Lyapunov exponent, and neighboring trajectories diverge from each other only algebraically in time (typically linearly in time). We have shown only a few representative tori; the regions they span are actually filled with a continuous family of tori. There are two main regions of tori, one in the region exterior to the main vortex, and one at the core of the recirculating region (shown in dark blue and green respectively). Sandwiched between the two is a "chaotic" or "stochastic" band, whose trajectory is shown in red. Trajectories in this region are 
chaotic, are characterized by a positive Lyapunov exponent (hence exponential divergence of trajectories), and fill out an apparently two-dimensional region. One also sees an elongated torus (light blue) embedded in the top of the chaotic zone. There appear to be a number of such tori of various shapes and sizes, though the small ones are hard to find. There is a symmetrically disposed stochastic band and region of "core" tori associated with the recirculating eddy on the upper boundary, but it is not shown.

Consider the behavior of an initially small blob of tracer located in a region of tori. The length of the cloud will grow algebraically (typically linearly) with time. Ultimately, the tracer will be redistributed uniformly along the invariant torus it is initially centered on, but in the absence of molecular diffusion there will be no mixing across tori. If a small molecular diffusion is introduced, it will act on the algebraically amplified cross-torus concentration gradients, resulting in greatly enhanced cross-torus diffusion. This is clearly a generalization of the shear dispersion phenomenon familiar from advection-diffusion problems involving steady flows.

Mixing in the chaotic zone will proceed very differently. Trajectories will separate exponentially rather than algebraically, leading to more efficient dispersal than possible among tori. More importantly, a small blob will ultimately become mixed over an area, rather than a curve, even in the absence of molecular diffusion. A typical example of such mixing is shown in Figure 3, which plots the time course of a cloud of 2000 particles located initially in a small disk within the chaotic zone. The disk is rather quickly elongated into a short segment, which then jumps around for several Poincare periods without being much further stretched. Then it encounters a region of rapid extension, and particle dispersion occurs in a burst. The geometry is still a filament, though, and mixing over a $2 \mathrm{D}$ region has not yet taken place. This occurs subsequently, through the stretching and folding of the filament, in a process reminiscent of the kneading of dough. Note that the folding and creation of multiple strands is an inevitable consequence of continued elongation of the tracer filaments, as folds must occur once the length of a filament exceeds the size of the domain.

The unstable manifold of $\mathrm{P}$ is the set of points whose Poincare orbits asymptote to $\mathrm{P}$ as time runs backwards; it is a key determinant of the mixing properties of the system. One can compute an approximation to the unstable manifold by numerically finding the fixed point of the map, and tracking the orbits of a small segment of particles initially 
located on a line coming out of the fixed point tangent to the unstable eigendirection. A segment of the unstable manifold, computed in this manner, is shown in Figure 4. There are clear similarities between the time course of mixing shown in Figure 3 and the geometry of the unstable manifold. The resemblance is particularly clear in the multiple stranded structure emanating from the vicinity of the left-hand fixed point of the Poincare map. It could be said that the tracer cloud initially wanders about without mixing much, until it "finds" the unstable manifold, whereafter it tracks it and consequently fills out the chaotic zone. We will see hints of the characteristic unstable manifold structure again when we consider vorticity mixing in the fully nonlinear equations in $\S 4$.

The extent of the chaotic zone is a characteristic of prime physical importance, as it determines the region over which efficient tracer homogeneization will occur. In Figure 5 ve show Poincare sections of the chaotic zone for various $\varepsilon$. For small $\varepsilon$ the chaotic zone closely hugs the heteroclinic streamline structure of the unperturbed flow. As $\varepsilon$ increases, the thickness of the zone increases roughly in proportion, until by $\varepsilon=.2$ there is no longer any hole in the center of the main eddy, and the tracer homogeneization is expected to become complete throughout the gyre. This suggests a strategy for identifying mixing regions in realistic geophysical flows. One would split up the flow into a "steady" (probably low frequency) and "fluctuating" part, use the former to identify heteroclinic structures, and use the latter to determine the approximate width of the associated stochastic zones.

Given that (2) is not actually a solution of the equations of motion, one must be wary of any results that depend on the detailed nature of the stipulated perturbation. Happily, chaos in such systems is very robust, and depends primarily on the unperturbed streamline geometry and the overall velocity amplitude of the perturbation. A typical example is shown in Figure 6, which displays a chaotic Poincare orbit for $\left(k_{2}, h_{2}\right)=(4,4)$ with $\varepsilon=.05$. This result (and many others like it we could show) suggests that the oversimplified specification of the perturbation is not a critical flaw.

\section{Characterization of the mixing: Correlation functions and spectra}

As illustrated in Figure 3, the mixing proceeds in two stages. In the first stage, the small blob of tracer is stretched out into a filament with size comparable to that of the chaotic zone. Thereafter the stretching and folding which leads to 
cross-streamline mixing commences. The stretching in the first stage is very intermittent in time, and can take quite a while to get underway. In fact, we have found the time required for this stage to be highly sensitive to the initial position of the blob, a property which is connected with the intricate and fine-grained spatial variability of finite-time estimates of the Lyapunov exponents. This matter, as it relates to chaotic mixing, is touched upon in Pierrehumbert 1990, and 1991a, but the issues are very deep and require much further investigation which is outside the scope of the present study.

We direct attention here to the second stage of mixing. How can we characterize the degree of mixedness during this stage? An obvious choice is the variance of the cloud of particles about its mean position. On reflection, this is found to be unsuitable. The variance already attains nearly its maximum value for a single filament snaking through the chaotic zone, at a stage when the flow is not at all well mixed. It is relatively insensitive to subsequent mixing.

Instead, we introduce the two particle correlation function $\mathrm{H}(\mathrm{r})$ as way to characterize mixing. Given a cloud of $\mathrm{N}$ particles, one computes $\mathrm{H}(\mathrm{r})$ in the following manner. First, compute the $\mathrm{N}(\mathrm{N}-1) / 2$ distances between all pairs of particles. $\mathrm{H}(\mathrm{r})$ is then defined as the number of pairs with distance less than $r . \quad H(r)$ encapsulates a great deal of valuable information about the geometry of the cloud. $\mathrm{H}$ asymptotes to a constant at large $r$, and the distance at which $\mathrm{H}$ begins to flatten is indicative of the overall extent of the cloud, be it a single strand or an area-filling tangle. Further, if $\mathrm{H}(\mathrm{r})$ exhibits a self-similar subrange in which $\mathrm{H} \sim \mathrm{r}^{\alpha}$, then $\alpha$ is the correlation dimension characterizing the geometry at the corresponding length scales (Grassberger \& Procaccia, 1983). $\alpha=0$ corresponds to particles clustered at a point, $\alpha=1$ corresponds to well-separated filaments of tracer, and $\alpha=2$ corresponds to an area-filling cloud; the whole panoply of intermediate structures associated with fractional $\alpha$ is also available.

Figure 7 hows the time evolution of the structure function for $\left(\mathrm{k}_{2}, \mathrm{l}_{2}\right)=(1,2)$ with $\varepsilon=.2$, plotted on logarithmic axes. The initial condition consists of 10000 particles in a square of side .01 centered on the point $(\pi / 2,25)$. Let $H_{n}(r)$ be the structure function after $n$ iterates of the Poincare map. For $\mathrm{n} \leq 5$ there is a single subrange of slope unity that extends from small scales out to where $\mathrm{H}_{n}$ begins to flatten out. This corresponds to the stage at which the cloud has been stretched out to a single filament, or perhaps a few very closely spaced filaments. For larger $n$, $\mathrm{H}_{\mathrm{n}}$ exhibits two distinct subranges - a slope- 1 subrange at 
small $r$ and a subrange with slope slightly less than 2 at somewhat larger $r$. The elbow demarcating the two regimes moves to ever smaller $r$ as $n$ increases. An arrangement of thin filaments more or less uniformly spaced is one geometry consistent with this behavior; the elbow of the correlation function corresponds to the mean spacing of the filaments. This picture is borne out by Figure 8, which shows a snapshot of the particle cloud at $n=7$. As with the Poincare cross sections, the behavior of the structure functions is not particularly sensitive to the detailed form of the perturbation. In Figure 9 ve show $\mathrm{H}_{\mathrm{n}}(\mathrm{r})$ for a $(4,4)$ perturbation with $\varepsilon=$ .2. It shows all the same features present in the previous case.

The mixing is very nonlocal. It does not proceed in a diffusive manner. Diffusion would eliminate small scale inhomogeneities first, and then spread the tracer gradually to fill the macroscopic vessel. In contrast, for chaotic advection $\mathrm{H}_{\mathrm{n}}(\mathrm{r})$ converges to its asymptotic value first at large $\mathrm{r}$ as $\mathrm{n}$ increases, and convergence spreads to smaller distances as time progresses. The mixing first distributes the tracer throughout the chaotic zone, in the coarse-grained sense. It then proceeds to eliminate fine-grained inhomogeneities. As the mixing continues, one must examine ever smaller scales in order to detect inhomogeneity. This kind of mixing is more like the shuffling of a deck of cards in which the top half is initially black and the bottom is initially white. The first shuffle distributes black cards throughout the thickness of the deck, though there are still many clumps of black cards in bands. Subsequent shuffles mix up the bands and give a more uniform distribution. "Eddy diffusivity" is unlikely to be a productive approach to parameterizing mixing by chaotic advection, but it is not clear what should take its place. The following remark provides some guidance, however. Suppose that the length of filaments increases exponentially in time with average rate $\lambda$ (related to the Lyapunov exponent, which in turn is related to the amplitude and fluctuation intensity of the large scale wave). Then, if the domain over which chaotic mixing takes place has a characteristic length scale $\mathrm{L}$, the typical strand spacing scales with $\mathrm{Le}^{-\lambda t}$ because a strand of length $\mathrm{e}^{\lambda t}$ must have roughly $\mathrm{L} / \mathrm{e}^{\lambda \mathrm{t}}$ folds if it is crumpled so as to fit into the domain. Hence, the time required for inhomogeneities to be eliminated down to scale $\mathrm{d}$ is proportional to $\lambda^{-1} \ln (\mathrm{L} / \mathrm{d})$, and this state of affairs occurs only after the tracer is distributed across the whole domain, in the coarse-grained sense. In contrast, the diffusive damping time for inhomogeneities of scale $d$ is $d^{2} / D$, and the (longer) time required for diffusive

Geophysical and Astrophysical Fluid Dynamics 58, 285-320. 
transport to fill out a region of size $\mathrm{L}$ is $\mathrm{L} 2 / \mathrm{D}$, where $\mathrm{D}$ is the diffusion coefficient.

It should be evident from the preceding discussion that $\mathrm{H}(\mathrm{r})$ contains much of the information traditionally obtained from spectral descriptions of the tracer pattern. In fact, elementary manipulations establish that the power spectrum of a cloud of delta-functions characterized by a structure function $\mathrm{H}(\mathrm{r})$ is

$$
C(\mathrm{k})=\text { (const.) } \mathrm{k} \int_{0}^{\infty} \frac{\mathrm{dH}}{\mathrm{dr}} \mathrm{J}_{0}(\mathrm{kr}) \mathrm{dr}
$$

where $\mathrm{J}_{0}$ is a Bessel function and $C(\mathrm{k})$ is the conventional isotropic concentration variance spectrum, defined such that its integral from $k=0$ to $\infty$ is the net variance of the tracer concentration. (See Pierrehumbert 1990 and Weiss and McWilliams 1990 ) An immediate consequence of (3) is that if $\mathrm{H}(\mathrm{r})$ has an extensive subrange with behavior $\mathrm{r}^{\alpha}$, then $C(\mathrm{k})$ will have a subrange with behavior $\mathrm{k}^{1-\alpha}$. This observation links the correlation dimension with the classical notion of self-similar (i.e. power-law) spectra. A more complete discussion of the connection between spectra and fractal geometry may be found in Pierrehumbert 1990 and Pierrehumbert 1991b. The spectra associated with fractal vorticity distributions range from $\mathrm{k}^{1}$ to $\mathrm{k}^{-1}$; steeper spectra do not have enough power at small scales to support a spatially self-similar geometry. An important subtlety, discussed in Pierrehumbert 1991b, is that the limits $\mathrm{k} \rightarrow \infty$ and $\alpha \rightarrow 2$ are not interchangeable. One recovers the $\mathrm{k}^{-1}$ spectrum at sufficiently short waves for $\alpha$ infinitesimally less than 2 , but if we set $\alpha=2$, the coefficient of the $\mathrm{k}^{-1}$ leading term of the asymptotics vanishes. This is so because $\int \mathrm{rJ}_{0}(\mathrm{kr}) \mathrm{dr}$ vanishes for nonzero $\mathrm{k}$, being the $2 \mathrm{D}$ Fourier transform of a constant. In this case the spectrum is steeper than $\mathrm{k}^{-1}$, the precise steepness being determined by the nature of the discontinuities of the vorticity field and its derivatives. The $\mathrm{k}^{-2}$ spectrum reported in Saffman (1971) is an example of this behavior, corresponding to patches of uniform vorticity demarcated by sharp gradients. We have found that for $\alpha=2-\delta$, the spectrum exhibits a steep spectrum at moderate $\mathrm{k}$ but a $\mathrm{k}^{-(1-\delta)}$ spectrum at sufficiently short waves.

The spectrum computed by Bessel transform of $\mathrm{H}_{20}(\mathrm{r})$ for the $(1,2)$ perturbation case is shown in Figure 10. In accordance with the almost two-dimensional mixed subrange shown in the correlation plots, the spectrum shows $\mathrm{a} \mathrm{k}^{-1}$ shortwave behavior. This is precisely the same as the classical spectrum of a passive tracer in homogeneous, 
isotropic 2D turbulence (see e.g. Rhines 1979). However, the classical reasoning relies on scaling arguments which build upon assumptions of locality in wavenumber space. For chaotic mixing, these arguments are invalid in their customarily stated form, as the shortwave spectrum is determined nonlocally, being controlled entirely by the large scale advection. Hence the replacement of the scaling argument with the geometric argument based on mixing to a dimension slightly below 2 is a critical step.

In point of fact, a simple modification to Kraichnan's (1971) phenomenological derivation of logarithmic corrections to the enstrophy-cascading spectrum also yields a $\mathrm{k}^{-1}$ spectrum for the tracer spectrum in chaotic advection. Paraphrasing Kraichnan's eqn. (1.5), if $\Lambda(\mathrm{k})$ is the net concentration variance transfer from all scales $<\mathrm{k}$ to all scales $>\mathrm{k}$, then

$$
\Lambda(\mathrm{k}) \sim \frac{\mathrm{k} C(\mathrm{k})}{\tau(\mathrm{k})}
$$

where $\tau$ is the characteristic time scale for distortion of concentration structures with scale $\mathrm{k}^{-1}$. Kraichnan proceeds to compute $\tau$ in terms of an integral over all scales of motion with wavenumbers less than $\mathrm{k}$, and then chooses $C$ so as to make $\Lambda$ constant, as required in an equilibrium situation. For chaotic advection, in contrast, the characteristic time for distortion of small concentration blobs is fixed by the separation rate of neighboring trajectories of the large scale advecting flow. In consequence, $\tau$ is independent of $\mathrm{k}$ and we require $C(\mathrm{k}) \sim \mathrm{k}^{-1}$. The situation is rather ironic: Kraichnan found a logarithmic correction to the spectrum that restores the integrity of the scaling argument by preserving locality. Perversely, by assuming sufficiently extreme nonlocality we obtain a scaling argument that recovers the uncorrected result.

\section{Vorticity mixing in fully nonlinear integrations}

Potential vorticity is also a tracer, but the key difference with a passive tracer is that, owing to self-induced rotation, small scale vorticity concentrations can resist being sheared out by the large scale flow field. However, if the large scale strain is sufficiently strong compared to the small scale vorticity, the latter may nevertheless be dispersed in a fashion qualitatively similar to the passive tracer dispersion illustrated above. The genericness of the mixing, noted above, is of importance in this context. As long as a vortex is not so intense as to hold together, the fact that its evolution alters the detailed form of the large scale advecting flow should not change the general properties of the mixing. 
These ideas are investigated through numerical integrations of the barotropic vorticity equation

$$
\partial_{\mathrm{t}} \nabla^{2} \psi+\mathrm{J}\left(\psi, \nabla^{2} \psi+\beta \mathrm{y}\right)=0
$$

subject to the initial condition

$$
\begin{aligned}
& \psi(\mathrm{x}, \mathrm{y}, 0)= \\
& \mathrm{c}_{1} \mathrm{y}+\mathrm{A} \cos \left(\mathrm{k}_{1} \mathrm{x}\right) \sin \left(\mathrm{l}_{1} \mathrm{y}\right)+\varepsilon \cos \left(\mathrm{k}_{2} \mathrm{x}-\delta\right) \sin \left(\mathrm{l}_{2} \mathrm{y}\right)
\end{aligned}
$$

With $\varepsilon=0$ the wave retains its form indefinitely in time, as it is an exact nonlinear solution; trivially, there is no mixing. The picture would be complicated by instability of the primary wave, but suppression of the subharmonic by the boundary conditions seems to eliminate such instabilities; the degree of geometric confinement is not sufficient to guarantee stability by Arnold's second theorem, however, and we are unaware of any proof of this evident stability. For nonzero $\varepsilon$ the initial condition is no longer a steady solution, and so there will be time fluctuations, which presumably increase in amplitude with increasing $\varepsilon$. The fluctuations of $\psi$ in this problem cannot be expected to resemble those in the constrained advection problem (2), so $\varepsilon$ plays a more complicated role here. An important difference from (2) is that the amplitude of the fluctuations need not be time independent. If the system approaches a steady state (through vorticity mixing or otherwise), the time fluctuations will decay to zero and further mixing will cease.
Nonetheless, we can look for the signature of chaotic advection in the course of this time evolution.

We could examine passive tracer mixing in the time varying $\psi$ field yielded by such an integration, but our main concern here is with dynamical implications of chaotic advection. Hence we will confine our analysis to the potential vorticity field associated with $\psi$ itself. One might think this would be a difficult system to analyze, in that the vorticity is providing its own stirring. However, because $\psi$ is related to the vorticity by an inverse Laplacian, it will have a larger characteristic scale than the vorticity, once small scale vorticity structures are generated. This scale separation means that vorticity and streamfunction can almost be regarded as separate entities. A particularly clear cut example of this is provided by nonlinear Rossby wave critical level dynamics (see Killworth \& McIntyre 1985 for a review), in which the vorticity field can be precisely regarded as being advected by the large scale component of the streamfunction alone.

A more serious problem with the chosen family of initial conditions is the dual role of $\varepsilon$ with regard to the survival of small vortices. Increasing $\varepsilon$ on the one hand favors survival of small vortices by increasing their vorticity; on the other hand it favors their destruction by increasing the 
magnitude of the fluctuation of the large scale flow. Hence, we do not address the question of formation and survival of small intense vortices such as studied by McWilliams (1984) in the case of homogeneous turbulence. Such vortices do not emerge, or play any role, in any of the calculations to be reported below.

The technology for numerical integration of (2) is well established, and little needs to be said about it. For historical reasons we employ a semi-spectral numerical model (pseudospectral in $\mathrm{x}$, with second-order finite differences in y). The timestep is a standard leapfrog, except for episodic employment of Euler-backward to keep the even and odd time levels from drifting apart. All calculations reported below were carried out with 512 mode resolution in $\mathrm{x}$ and 256 gridpoints in $\mathrm{y}$. There is no diffusion in the calculation, except for what may have been introduced numerically. None of the results presented are limited by the resolution. At higher resolution, one can track the vorticity field farther in time before Gibbs' phenomena interfere, and hence one can see the vorticity mix down to finer scales. However, the resolution employed is well in excess of that needed to isolate the basic behavior. There is no indication of anything fundamentally new happening with increased resolution in this series of experiments.
In this section we shall discuss three simulations. Experiment [1x2] was done with $\mathrm{k}_{2}=1$ and $\mathrm{l}_{2}=2, \varepsilon=.2$ and $\delta=0$. It is indicative of the mixing proceeding from a flow with initial vorticity entirely in the largest scales. Experiment $[1 \times 2 s]$ is precisely the same, except that the perturbation is shifted by a quarter wave with respect to the basic state wave $(\delta=\pi / 2)$. It provides an interesting comparison with Experiment [1x2], as the initial conditions have precisely the same energy and enstrophy, yet lead to very different evolution. Experiment [4x4] was conducted with $\mathrm{k}_{2}=\mathrm{l}_{2}=4$, $\varepsilon=.1$ and $\delta=0$, and is indicative of the behavior when there is an initial scale separation between the basic state wave and the vorticity perturbation.

The vorticity evolution for Experiment [1x2] is shown in Figure 11. In this experiment, the initial perturbation has extrema in phase with those of the basic state; it strengthens the vortex at the upper right of the domain and weakens the vortex at the lower left. The signature of chaotic mixing is very clear in this case. The weakened vorticity of the lower left vortex is stretched and folded into a series of ever finer and more closely spaced filaments, and its variation is thus cascaded down to ever smaller scales. The vorticity in the open streamline region exterior to the gyre, where we found tori in the passive 
advection case, undulates but does not show similar signs of mixing. With the grey-scale palette employed in Figure 11 it is difficult to see what is happening to the vortex at the upper right. Hence, we show this region with a remapped palette in Figure 12. It is evident that this vortex shows a "core" region which does not homogenize or mix with the surrounding fluid, surrounded by a relatively thin mixed (chaotic) band. This behavior is consonant with the $\varepsilon$ dependence of the stochastic zone shown in Figure 5. The upper vortex is stronger relative to the magnitude of the time fluctuations, and so has a chaotic zone that more closely hugs the unperturbed heteroclinic cycle.

The vorticity fields for Experiment [1x2s] are shown in Figure 13. In this case the initial perturbation does not particularly favor one gyre over the other. In this case, both large scale gyres have a well-defined core surrounded by a broad stochastic band. An interesting feature of this experiment is the persistent transient pulsation of the core regions. The unmixed core vorticity attains a vaguely elliptical shape, which undergoes repeated elongation and contraction as it rotates. The pattern is already evident at $\mathrm{t}=39$, and while there is some indication of slow relaxation to a steady state, the fluctuations are still considerable at $\mathrm{t}=63$. This behavior is reminiscent of the Great Dark Spot of
Neptune, which has been interpreted as a pulsating Kida vortex by Polvani et al. (1990). The contrast between Experiment [1x2] and Experiment [1x2s] shows that whether one gets a steady core (like Jupiter's Red Spot) or a pulsating core (like Neptune's Dark Spot) can be a matter of initial conditions. That radically different end states can evolve from initial states [1x2] and [1x2s] having identical energy and enstrophy has some interesting consequences for the role of higher invariants in the statistical mechanics of $2 \mathrm{D}$ turbulence. We shall return to this point in $\S 6$.

Finally, we turn to Experiment [4x4], shown in Figure 14. This is a particularly stringent test of the chaotic advection hypothesis, as the rms velocity amplitude of the perturbing small scale field is fully $33 \%$ of that of the large scale wave. The background wave is hardly detectable in the initial total vorticity field. Yet the large scale wave is there the whole time, controlling the vorticity mixing, and its form clearly emerges as the perturbation vorticity is mixed down to small scales. The end state is characterized by moderatesized vortex cores embedded in a broad stochastic band. The core vorticity is comparable in magnitude to the initial vorticity in the core region; a vortex present in the initial perturbation has become trapped in the region of core tori, strengthening the circulation and thus enhancing the 
predisposition of the large scale flow to have tori here. These cores do not exhibit pulsations like those observed in the previous case.

Vorticity cross sections along two lines of constant $\mathrm{x}$ intersecting the two large scale gyres are shown for each case in Figure 15. Case [1x2] clearly shows the broad homogenized region associated with the lower left eddy. There is no unmixed core, and the gradients are expelled to the exterior of the eddy. The upper right eddy exhibits a core region as well as a (relatively thin) chaotic band, and region of exterior gradients. In Case [1x2s] the structure of the two vortices is less asymmetric. Both exhibit a core vortex region of approximately the same size, surrounded by a homogenized region, which in turn is bounded by an exterior region of strong gradients. Case [4x4] yields a similar pattern, though the exterior gradient regions are somewhat less prominent visually, owing to the higher vorticity variance (which stems from the initial conditions).

The long term behavior in all these cases may be summed up as follows. The flow evolves to a nearly steady state (in the coarse grained sense), with scales and streamlines resembling those of the initial large scale wave, but having redistributed vorticity. The end state vorticity distribution consists of regions of homogenized vorticity surrounding the heteroclinic structures of the large scale wave, and regions of smooth vorticity gradients corresponding to regions of tori in the associated chaotic advection problem. The gradient-containing regions are of two types: one is associated with the layer of "open" streamlines snaking between the two recirculating gyres of the large scale flow. The other (which is present only if the time variability is sufficiently weak) is associated with the core region of the recirculating gyres. The former represents a barrier to mixing analogous to that found at the edge of a simulated polar vortex in the laboratory experiments of Sommeria, Meyers \& Swinney (1988). The appearance of invariant tori in periodically perturbed Hamiltonian flow is a familiar phenomenon, but their survival in the face of the broadband time fluctuations appearing in the vorticity simulations is striking and unexpected.

The formation of ever finer grained vorticity filaments as time progresses is an instantiation of enstrophy cascade to small scales. The mixing is highly nonlocal in wavenumber space, and so invalidates the classical similarity arguments leading to the Kolmogorov-Kraichnan spectrum. However, the resemblance of the vorticity evolution to passive tracer mixing by chaotic advection leads one to conjecture that a $\mathrm{k}^{-1}$ shortwave spectrum may nevertheless 
emerge, as it does in the passive tracer case. A missing link in this argument is that the reasoning summarized in $\S 3$ connecting geometry to spectra is based on clouds of vortices of identical strength, whereas the actual vorticity fields have continuously distributed value. This may not be a critical failing, as from a coarse-grained perspective regions of large vorticity can be approximated as regions of high density of vortex blobs, and conversely. At this point, however, we are not certain how to make this argument more rigorous.

The enstrophy spectra for experiments [1x2s] and [4x4] are shown in Figure 16. These are isotropic spectra, angle-averaged over all wavenumbers with a given magnitude, and multiplied by $\mathrm{k}$ so that the integral with respect to $\mathrm{k}$ gives the total enstrophy. The wavenumber is normalized such that $\mathrm{k}=1$ corresponds to the length of the gravest non-constant mode in $\mathrm{x}$. As is evident from the vorticity cross sections in Figure 15, the vorticity is not periodic in y (owing to the beta effect). If left in, this would itself lead to a $\mathrm{k}^{-2}$ contribution to the enstrophy spectrum. Hence we have detrended the vorticity field by subtracting off $\beta *$ y before computing spectra.

In both cases, the spectra rather quickly exhibit a self-similar range at small scales (wavenumbers 10-100).
When it first appears, the slope is steep, the shape being roughly $\mathrm{k}^{-2}$. In accordance with the arguments of Saffman (1971), this is indicative of an arrangement of well-separated curves on which the vorticity gradient is large, separating the plane into regions within which the vorticity is comparatively uniform. As time progresses, the slope decreases, and ultimately approaches the $\mathrm{k}^{-1}$ classical form. The classical spectrum emerges more quickly in experiment $[4 \times 4]$ than in experiment $[1 \times 2 s]$. The time course of the spectrum is precisely opposite to that seen in experiments such as McWilliams (1984), Benzi et al. (1986) and Legras et al. (1988) beginning with spatially homogeneous and isotropic initial conditions; in these cases the spectrum is initially shallow, and becomes progressively steeper as time goes on and small coherent vortices freeze out. Though our experiments reveal the same dichotomy between steep and classical spectra, the physics of what is going on is completely different from the homogeneous case. In the experiments discussed here, small scale persistent vortices never appear, presumably because the large scale strain is too strong to allow them to survive. The initial steep spectra are instead associated with a certain degree of smoothness in the filamentary structure, which is lost as time goes on.

Geophysical and Astrophysical Fluid Dynamics 58, 285-320. 
Our discussion to this point has emphasized the emergence of a classical shortwave spectrum. However, it should be noted that in the present problem (and perhaps more generally) this spectral range is characterizing a part of the flow which is dynamically rather uninteresting and perhaps entirely irrelevant. It is telling us something about the "fuzz" on the vorticity curves in Fig 15, whereas the overall shape of the curves is clearly more important. The spectrum in the range $\mathrm{k}=10$ to 100 accounts for around $1 \%$ of the total enstrophy at the later times. What is far more interesting is that the classical spectrum begins at about $\mathrm{k}=10$. Recall that these experiments represent decaying turbulence, rather than continuosly forced turbulence, so there is no injection length scale to naturally determine the boundary of the enstrophy cascading subrange. There is no obvious reason the enstrophy cascading region shouldn't extend all the way to the largest scales, instead of stopping an order of magnitude short. That it doesn't is indicative of the stability of some large scale flow "nearby" (in a function theoretic sense) to the initial conditions. This hypothetical flow is resistant to disruption, and so its enstrophy cannot be bled off to small scales.

The structure of the large scale spectrum is itself rather revealing. In the wavenumber range 1-10, it is steep but not exponentially so; the behavior here is suggestive of a $\mathrm{k}^{-3}$ to $\mathrm{k}^{-4}$ enstrophy range, though given the discreteness at such long waves it is not possible to be very precise. An infinitely smooth (infinitely differentiable) vorticity distribution would yield an exponentially decaying spectrum, so the algebraic spectrum indicates that, viewed at large scales, the vorticity field exhibits jumps (more precisely, rapid transitions) in sufficiently high order derivatives. It is noteworthy that a jump in vorticity gradient yields a $\mathrm{k}^{-4}$ isotropic enstrophy spectrum. This picture is consistent with the rather sharp boundaries between macroscopic homogenized regions and non-homogenized regions evident in Figures 12-14 and Fig 15.

The following overall picture emerges. The crossover wavenumber represents the intersection of the steep spectrum characterizing a stable large-scale eddy with the $\mathrm{k}^{-1}$ spectrum characterizing the chaotically mixing, enstrophy-cascading, part of the vorticity. Since the enstrophy is constant (and finite) at all times, the $\mathrm{k}^{-1}$ subrange must have a shortwave cutoff $\mathrm{k}_{0}$, which increases with time as the mixing proceeds to ever finer scales. If the enstrophy range is written as $\mathrm{A}(\mathrm{t}) \mathrm{k}^{-1}$, then $\mathrm{A}$ must decay like $1 / \log \left(\mathrm{k}_{0}\right)$, in order to keep the enstrophy in this range constant. Hence, the crossover wavenumber increases with 
time and eventually disappears from view, leaving only the large eddy behind. Viewed in this way, the initial condition projects (nonlinearly) on a "steady state" plus a "perturbation" vorticity field, and as time goes on the enstrophy cascade acts as a dissipation, getting rid of the latter by removing it to small scales. The details of how this scenario is played out depends on the proximity of stable large scale states to the initial condition, and so is expected to be highly dependent on the configuration of the experiment at large scales.

\section{Vorticity mixing and upscale energy transfer}

The previous section established a connection between chaotic mixing, potential vorticity homogenization, and the downscale enstrophy cascade. We now examine the implications for the upscale energy tranfers that are the hallmark of two dimensional turbulence. The basic idea is as follows. If a small scale eddy is not strong enough to resist dispersal by the large scale flow, its vorticity will be redistributed over a large area. A small scale vortex loses energy in the course of being dispersed. Since energy is conserved for the system as a whole, the energy lost by the small scales must be gained by the large scales.
In order to formalize this idea, divide the streamfunction field up into a part $\Psi(\mathrm{x}, \mathrm{y}, \mathrm{t})$ which has large spatial scale, and $\psi^{\prime}(\mathrm{x}, \mathrm{y}, \mathrm{t})$ which is the advected perturbation field, having a small spatial scale. The former is the specified large scale field doing the advecting. Let the corresponding vorticity fields be Q and q'. We will show that the time-integrated Reynold's stresses acting on the large scale flow yield a net increase in energy when the passively advected field loses energy. The vorticity equation is

$$
\begin{aligned}
& \partial_{\mathrm{t}} \mathrm{Q}+\left[\partial_{\mathrm{t}} \mathrm{q}^{\prime}+\mathrm{J}\left(\Psi, \mathrm{q}^{\prime}\right)\right]+ \\
& \mathrm{J}\left(\psi^{\prime}, \mathrm{Q}\right)+\mathrm{J}(\Psi, \mathrm{Q})+\mathrm{J}\left(\psi^{\prime}, \mathrm{q}^{\prime}\right)=0
\end{aligned}
$$

However, if the small scale field is passively advected by the large,we have

$$
\partial_{\mathrm{t}} \mathrm{q}^{\prime}+\mathrm{J}\left(\Psi, \mathrm{q}^{\prime}\right)=0
$$

and the bracketed terms in (6) vanish. To form the large scale energy budget, we multiply (6) by $\Psi$ and integrate over all space. Denoting the spatial average by <·>, and using integration by parts, we find that

$$
-\frac{\mathrm{dE}}{\mathrm{dt}}+\left\langle\Psi \mathrm{J}\left(\psi^{\prime}, \mathrm{Q}\right)\right\rangle+\left\langle\Psi \mathrm{J}\left(\psi^{\prime}, \mathrm{q}^{\prime}\right)\right\rangle=0
$$

where $\mathrm{E}$ is the kinetic energy of the large scale flow. The first averaged term is linear in the primed quantity and therefore will nearly vanish if there is a separation in scale 
between the primed and unprimed quantities. Thus, within this approximation

$$
\frac{\mathrm{dE}}{\mathrm{dt}}-\left\langle\Psi \mathrm{J}\left(\psi^{\prime}, \mathrm{q}^{\prime}\right)\right\rangle=0
$$

The bracketed expression is the conventional conversion term appearing in most diagnostic analyses of atmospheric energy budgets. Further, by multiplying the passive advection equation by $\psi^{\prime}$ and integrating, we find that

$$
-\frac{\mathrm{dE}^{\prime}}{\mathrm{dt}}-\left\langle\Psi \mathrm{J}\left(\psi^{\prime}, \mathrm{q}^{\prime}\right)\right\rangle=0
$$

where $E^{\prime}$ is the kinetic energy of the primed field. In consequence $\frac{\mathrm{d}\left(\mathrm{E}+\mathrm{E}^{\prime}\right)}{\mathrm{dt}}=0$, and we have the desired result than any energy lost by the small scale eddies is gained by the large scale.

Now let us see how well these ideas work in vorticity simulations such as discussed in $\S 4$. Since the eddies of interest are large scale and anisotropic, we have found it most informative to decompose the motion into Fourier modes in $\mathrm{x}$ alone, aggregating all modes with zonal wavenumber $n$ into the energy $E_{n}(t)$. Figure 17 hows the behavior of the energy for $A=1$ subject to a $(4,4)$ perturbation with $\varepsilon=.2$. As predicted, the fundamental mode energy $E_{1}$ increases in step with the decay of the perturbation energy $\mathrm{E}_{4}$. The effect of varying $\varepsilon$ and $\mathrm{A}$ is shown in Figure 18. Focusing first on the results at fixed $\mathrm{A}$, we see that the time scale for the energy capture is insensitive to $\varepsilon$. This observation establishes that the energy transfer is not due to subharmonic instability of the $(4,4)$ wave, whose growth rate would scale with $\varepsilon$. Reducing $A$, on the other hand, does delay the energy capture.

The above results establish that the large eddy serves as the pacemaker for the upscale energy transfer, and hence points the way to a simple parameterization of large eddy maintainence. However, it is fair to point out that the capture of energy by the large scale is virtually complete by $\mathrm{t}=2$, a stage well before chaotic mixing of the vorticity has gotten underway. At this stage, the small scale eddies have become substantially elongated, but the filaments have not yet completed a single circuit about the large eddies. Chaotic mixing of vorticity in principal could be a potent means of cannibalizing the small eddy energy, but the effect is clearly not of importance in the experiments reported here; this is underscored by the fact that the behavior in Figure 18 shows no qualitative change as $\mathrm{A}$ is reduced below .5 and the heteroclinic cycle is eliminated.

What is going on here is really not so very different from the energy capture mechanism discussed by Shutts 
(1983) in connection with maintainence of atmospheric blocking patterns by synoptic transient eddies. Shutts noted that the shearing out of small eddies by a large scale diffluent jet creates Reynolds' stresses which pump energy into the large scale, an effect which was already well known in the simpler context of plane Couette flow. Haines and Marshall (1987) indeed found such energy transfers to manifest themselves in fully nonlinear simulations of small eddies propagating through blocking patterns.

Blocking presents a natural arena for the application of chaotic advection concepts, as blocking is practically synonymous with the appearance of closed streamlines, particularly at lower levels. As noted above, nothing fundamentally new has been learned with regard to the energy budget of the block. The novel implications concern the potential vorticity budget. One expected effect is the homogenization of potential vorticity within the blocking region; this is unlikely to seriously affect the persistence of the block. An effect of greater import is the exchange of potential vorticity between the block and its surroundings, via leakage through thin tails like those appearing at the bottom boundary of Figures 4 and 8 . This interchange would erode the potential vorticity of the block, which cannot in any obvious way be resupplied by interactions with the synoptic eddy field. One would need a mechanism for preferential ingestion of potential vorticity anomalies of a given sign.

It is noteworthy that the upscale energy transfer discussed in this section occurs without benefit of either vortex pairing or a $\mathrm{k}^{-5 / 3}$ energy cascading inertial subrange. We suggest that the situation presented above - in which there is an energetic planetary wave that directly captures energy of any small eddies which appear and then proceeds to mix their enstrophy away to small scales - is a better archetype for turbulent flow in the Atmosphere than the traditional homogeneous picture based on an injection range separating longwave energy cascade and shortwave enstrophy cascade. Indeed, Boer and Shepherd (1983) found that the Atmosphere exhibits an enstrophy cascading inertial range at small scales, but that the energy cascading inertial range spectrum is missing, even though there are clear transfers of energy to the largest scales of motion.

\section{Discussion and conclusions}

Using modulated traveling Rossby waves as an example, we have investigated the properties of mixing by class of large scale two-dimensional flows of geophysical interest. In these flows, which are distinguished by regions 
of recirculating streamlines bounded by a separatrix, enhanced mixing occurs in a stochastic band surrounding the separatrix. The character of the mixing, as revealed by an analysis of the two-particle correlation function, is different from diffusion. It first mixes the tracer over a broad area in the coarse grained sense, and then proceeds to eliminate progressively finer grained inhomogeneities. This kind of nonlocal (in physical space) mixing cannot be faithfully represented as an eddy diffusion, and insofar as similar phenomena occur in nature, attempts to characterize large scale mixing by eddy diffusion coefficients derived from data are bound to give unreliable results.

Even though the mixing is governed by processes that are very nonlocal in wavenumber space, the power spectrum of passive tracer concentration obeys a classical $\mathrm{k}^{-1}$ form. We have obtained this result from two disparate arguments. The first is a geometric argument based on fractal dimension of the concentration distribution. The second is a phenomenological scaling argument based on scaleindependence of the characteristic time for distortion of concentration blobs.

Integrations of the barotropic vorticity equation subject to initial conditions consisting of a perturbed large scale Rossby wave demonstrate that the chaotic advection ideas have considerable explanatory power when applied to potential vorticity mixing. Homogenized vorticity regions appear where one expects chaotic zones in the corresponding passive advection problem, while regions of surviving potential vorticity gradients correspond to regions of tori in the advection problem. To make the theory fully predictive, one would need a means of estimating the amplitude of large scale temporal fluctuations proceeding from a given initial condition; we have not yet accomplished this.

Vorticity mixing by chaotic advection provides a clear-cut example of macroscopically irreversible mixing produced by the microscopically reversible Euler equations. Hopefully, this remark will resolve any remaining controversy surrounding the use of the term "Rossby wavebreaking" by McIntyre and Palmer (1983). The controversy (which in our opinion is largely pointless) focuses on the question of whether the resulting mixing is reversible (McIntyre and Palmer 1985). Chaotic mixing is macroscopically irreversible despite the reversible Euler equations in precisely the same sense that molecular diffusion is irreversible despite the reversibility of Newtonian mechanics. Strictly speaking, if one runs the system backwards, the mixed cloud in Figure 8 will condense back into a small blob, "unscrambling the egg," as 
it were. However, owing to the exponentially sensitive dependence of trajectories on initial conditions, a very small perturbation to the flow or the particle positions would prevent the unscrambling.

Considered as a decaying 2D turbulence experiment, our results have some interesting implications for the nature of the enstrophy cascading subrange in the presence of an energetic large scale wave. In the cases we have considered, the large eddy controls the enstrophy transfer at arbitrarily small scales, with local (in wavenumber space) interactions playing no detectable role. Despite the extreme nonlocality, a classical $\mathrm{k}^{-1}$ enstrophy spectrum ultimately emerges at small scales. Given the manifest similarity between vorticity mixing and passive tracer mixing in these experiments, the enstrophy spectrum is probably accounted for by arguments similar to those we have advanced for the passive tracer case. The vorticity participating in this spectrum is "fossil vorticity," which has already given up most of its energy to the large scale waves; upscale energy transfer acting to maintain the planetary wave does indeed occur in our experiments, but it is essentially complete after a single large-eddy turnover time. The energy transfer is associated with the initial distortion of the small scale eddy field, and is independent of chaotic vorticity mixing.
The importance of the large eddy should not be too surprising, in light of the well-known theoretical arguments for nonlocality of the enstrophy cascading inertial range. Kraichnan (1971) gets around the nonlocality by finding logarithmic corrections that restore locality; our findings show that Nature has at least one other way out of the dilemma. Speculations about the connection with 2D turbulence in the Atmosphere are premature at this stage, but our results demonstrate that the effect of the planetary waves must be a central concern in any study of this phenomenon. Indeed, Shepherd (1987a), who seems to have been the only investigator to have worried about such matters previously, found that half of the spectral enstrophy transfer was associated with interactions between the large scale stationary flow and the small eddies. He suggested that this was due to shearing out of eddies by the zonal flow. If one enlarged consideration to include low frequency fluctuation of the wavy planetary jets, chaotic advection comes into play and one may be able to account for even more of the enstrophy transfer. The question is whether most of the enstrophy transfer to small scales occurs in regions of tori or regions of chaos; this is an empirical question, that can be answered with reference to suitable analyses of the data. 
Our experiments, like those of Marcus (1988), show that if there is a "nearby" stable large scale state it tends to emerge, and the enstrophy cascade acts as a dissipation to get rid of the unwanted enstrophy (getting rid of unwanted initial energy is more problematic). Comparison of cases [1x2] and [1x2s] in $\$ 6$ demonstrates that knowledge of the initial energy and enstrophy alone does not suffice to determine the long term behavior of the system; it is a clear affront to formulations of statistical mechanics of 2D flow based on Kraichnan (1975). However, the two initial conditions do differ in their higher order invariants (like maximum and minimum vorticity). Recently, Miller (1990) has shown how to incorporate the full hierarchy of vorticity invariants into the statistical mechanics. Shepherd (1987b) demonstrated non-ergodicity on the energy-enstrophy hypersurface, based on existence of nonlinearly stable equilibrium states. This criticism doesn't apply to Miller's formulation, provided there is only a single stable state on an isovortical sheet; what Miller considers is essentially an excited state of such a vortex.

The output of Miller's theory is a vorticity field representing the ensemble mean (and presumably time mean) long term behavior. The vorticity in this state is always constant on the corresponding streamlines; it is an exact nonlinear solution of the Euler equations, and hence the fluctuations have no long term effect. The vanishing of the fluctuations is related to Miller's finding that mean field theory is exact for this problem, which is indeed why he is able to compute a solution. The vorticity evolution in our simulations appears consistent with the picture emerging from Miller's work, though the ultimate test of consistency would be to use Miller's formalism to compute the end-state corresponding to our initial conditions. This is a nontrivial and difficult computation, which must be deferred to future work.

Chaotic mixing is a productive and powerful concept which is likely to find many future applications in the atmospheric and oceanic sciences. Of the various possibilities, two problems are particularly ripe for study as they have an asymptotic regime in which potential vorticity exactly acts as a passive scalar. The problems are Rossby wave critical level dynamics (Killworth \& McIntyre 1985) and baroclinic equilibration with weak surface flow (Warn \& Gauthier, 1989). Both cases have been worked out for a single wave, which yields a steady advecting streamfunction; the ambient vorticity gradient is mixed algebraically by being wrapped up into a spiral. In both cases the steady streamfunction has a heteroclinic structure, and so will 
produce chaotic mixing if perturbed by a second wave. Given the generic nature of chaotic mixing, there is reason to hope that the fundamental behavior in these problems does not change much as the parameter controlling the asymptotics is made larger. Carrying out this program would build a bridge between the idealized behavior and real-world problems such as the dynamics of the equatorial stratospheric "surf zone" (McIntyre and Palmer 1983), or baroclinic equilibration in the presence of multiple unstable waves.

\section{Acknowledgements}

I never had the opportunity to discuss this work with Lee-Or Merkine (no doubt it would have been a better paper if I had), but I would like to acknowledge my debt to Lee-Or for many profitable and companionable interactions I have had with him over the years. His work on absolute instability, on flow over mountains, and on atmospheric blocking was an inspiration to me early in my career, when I was trying to get launched in the area of geophysical fluid dynamics. Lee-Or was a good colleague, and a good friend, and I shall sorely miss his presence.

I would also like to acknowledge the hospitality of the Meteorologiska Institutionen of Stockholms Universitet, and the Institute for Theoretical Physics of U.C. Santa Barbara, where some of this work was carried out. I am also grateful to Mr. Andrew Belmonte who assisted me with some exploratory passive tracer calculations for the modulated Rossby wave while he was an undergraduate intern in my group during the summer of 1987. The code used for numerical simulations of the barotropic vorticity equations was provided by Dr. Jeffrey Anderson. This work was supported by the National Science Foundation, under grant ATM 89-20589. 


\section{References}

Aref, H. 1984: Stirring by chaotic advection. J. Fluid Mech 143,1-21.

Benzi, R. , Paladin, G. , Patarnello, S. , Santangelo, P. and Vulpiani, A. 1986: Intermittency and coherent structures in two dimensional turbulence. J Phys. A 19 3771-3784.

Boer, G.J. \& Shepherd,T. 1983: Large-scale two dimensional turbulence in the atmosphere. J. Atmos. Sci 40,164-184.

Chernikov, A.A., Sagdeev, R. Z., and Zaslavsky, G. M. 1988: Chaos: How regular can it be? Physics Today 41 27-35.

Chien, W.-L, Rising, H. and Ottino, J.M. 1986: Laminar mixing and chaotic mixing in several cavity flows. J. Fluid Mech 170, 355-377.
Grassberger, P. and Procaccia, I. 1983: Measuring the strangeness of strange attractors. Physica 9D, 189208.

Guckenheimer, J. and Holmes, P. 1983: Nonlinear oscillations, dynamical systems and bifurcations of vector fields. Springer Verlag, New York, 453pp.

Haines, K. and Marshall, J. 1987: Eddy-forced coherent structures as a prototype of atmospheric blocking. Q. J. R. Meteorol. Soc., 113,681-704.

Hoskins, B. J., McIntyre, M.E. and Robertson, A. W. 1985: On the use and significance of isentropic potential-vorticity maps.Q. J. R. Meteorol. Soc., 111,877-946.

Khakhar, D. V., Rising, H. and Ottino, J.M. 1986: Analysis of chaotic mixing in two model systems. $J$. Fluid Mech 172, 419-451.

Killworth, P. and McIntyre, M.E. 1985: Do Rossby wave critical layers absorb, reflect or overreflect? J. Fluid Mech 161, 449 - 492. 
Knobloch, E. and Weiss, J.B. 1987: Chaotic advection by modulated traveling waves. Phys. Rev. A $\mathbf{3 6}$, 1522-1524.

Kraichnan, R. 1971: Inertial-range transfer in two- and three-dimensional turbulence. J. Fluid Mech 47, $525-535$

Kraichnan, R. H. 1975: Statistical dynamics of twodimensional flows. J. Fluid Mech. 67,155-175.

Legras, B., Santangelo, P. and Benzi, R. 1988: Highresolution numerical experiments for forced twodimensional turbulence. Europhys. Lett. 5, 37-42.

Marcus, P. S. 1988: Numerical Simulation of Jupiter's Great Red Spot. Nature 331 693-696.

McIntyre, M.E. and Palmer, T. N. 1985: A Note on the general concept of wave breaking for Rossby and gravity waves. Pageoph, 123, 964-975.

McIntyre, M.E. and Palmer, T.N. 1983: Breaking planetary waves in the stratosphere. Nature,305,593-600.
McWilliams, J. 1984: The emergence of isolated coherent vortices in turbulent flow. J. Fluid Mech 146, 2143.

Miller, J. 1990: Statistical Mechanics of Euler Equations in Two Dimensions. Phys. Rev. Letters, 65,21372140.

Ottino, J. M., Leong, C.W., Rising, H. and Swanson, P.D. 1988: Morphological structures produced by mixing in chaotic flows. Nature 333, 419-425.

Ottino, J. 1989: The kinematics of mixing: Stretching, Chaos and Transport. Cambridge University Press, Cambridge.

Pierrehumbert, R. T. 1990: Dimensions of Atmospheric Variability. in Searching for Certainty:Randomness, Prediction and Explanation in Modern Science, ed. J. Casti . CRC Press, in press.

Pierrehumbert 1991a: Large scale horizontal mixing in planetary atmospheres. Phys. Fluids, submitted. 
Pierrehumbert, R. T. 1991b: Geometrical considerations in two dimensional turbulence. in Nonlinear processes in atmospheres and oceans, eds. R. T. Pierrehumbert and G. Carnevale. Springer-Verlag, to appear.

Polvani, L. ,Wisdom, J. DeJong, E. \& Ingersoll, A.P.. 1990: Simple dynamical models of Neptune's Great Dark Spot.Science 249,1393-1397.

Rhines, P.B. and Young, W. R. 1982: Homogenization of potential vorticity in planetary gyres. J. Fluid Mech. 122,347-367.

Rom-Kedar, V., Leonard, A. and Wiggins, S. 1990: An analytical study of transport, mixing and chaos in an unsteady vortical flow. J. Fluid Mech 214, 347 394.

Rhines, P.B. 1979: Geostrophic Turbulence. Ann. Rev. Fluid Mech. 11 401-442.

Saffman, P. G. 1971: On the spectrum and decay of random two-dimensional vorticity distributions at large Reynolds number Stud. Appl. Math. 50377 383.
Shepherd, T. 1987a: A spectral view of nonlinear fluxes and stationary-transient interaction in the atmosphere. $J$. Atmos. Sci 44, 1166-1178.

Shepherd, T. 1987b Non-ergodicity of inviscid twodimensional flow on a beta-plane and on the surface of a rotating sphere. J. Fluid Mech 184, 289-302.

Shutts, G. J. 1983: The propagation of eddies in diffluent jetstreams: Eddy vorticity forcing of blocking flow fields. Q. J. R. Meteorol. Soc. 109, 737-761

Sommeria, J., Meyers, S.D., and Swinney, H.L. 1988: Laboratory model of a planetary eastward jet. Nature 337, 58-61.

Warn, T. and Gauthier, P. 1989: Potential vorticity mixing by marginally unstable baroclinic disturbances. Tellus41A, 115-131.

Weiss, J.B. and Knobloch, E. 1989: Mass transport by modulated traveling waves. Phys Rev. A 40, 25792589. 
Weiss, J.B. and McWilliams, J.C. 1990: Non-ergodicity of point vortices. J. Fluid Mech submitted.

Welander, P. 1955: Studies on the general development of motion in a two-dimensional, ideal fluid. Tellus $\mathbf{7}$, 141-156.

Wiggins, S. 1988: Stirred but not mixed. Nature 333, 395-396. 
Figures 


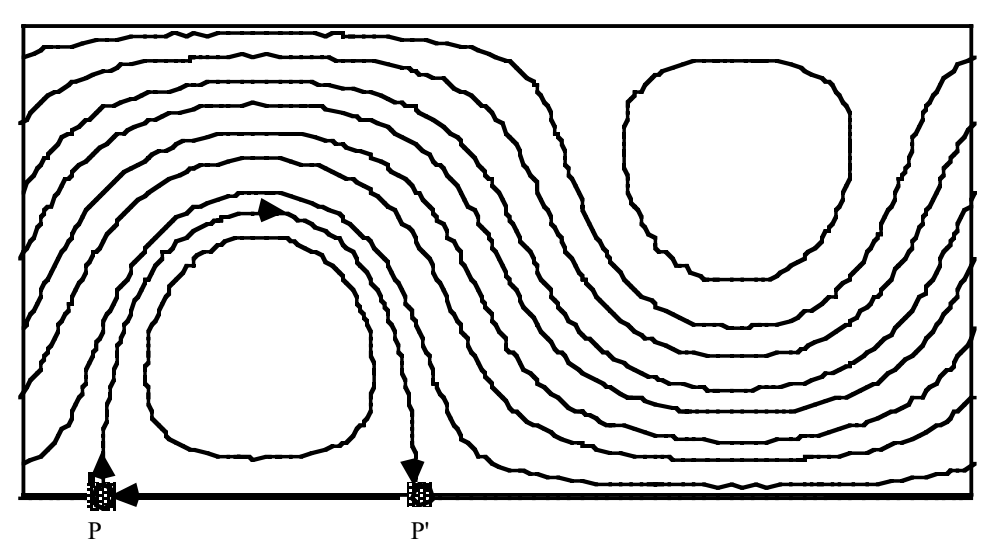

Figure 1........... Streamlines of basic state in the comoving reference frame for $\mathrm{A}=1$. $\mathrm{P}$ and $\mathrm{P}^{\prime}$ are fixed points of the unperturbed flow, and the heteroclinic cycle is emphasized.

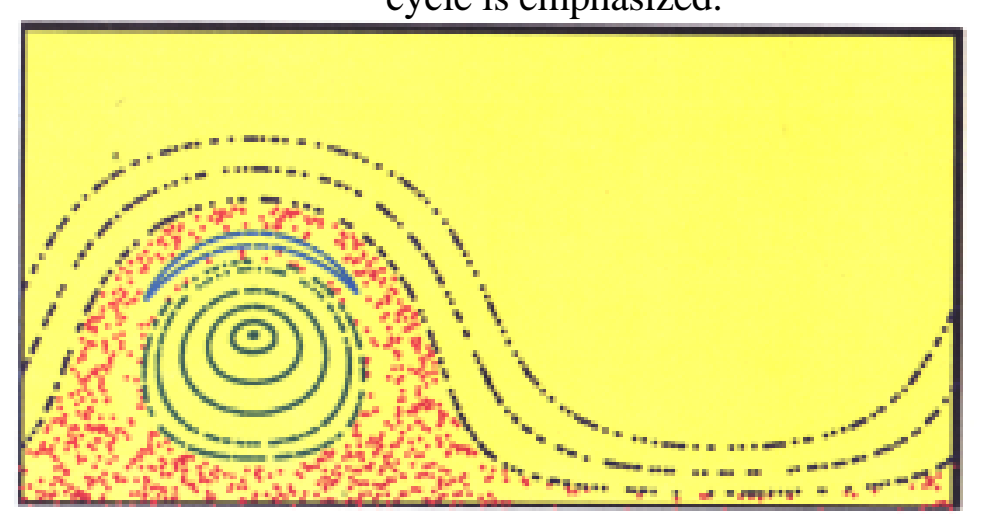

Figure 2 . Tori and chaotic regions for $\varepsilon=.1$
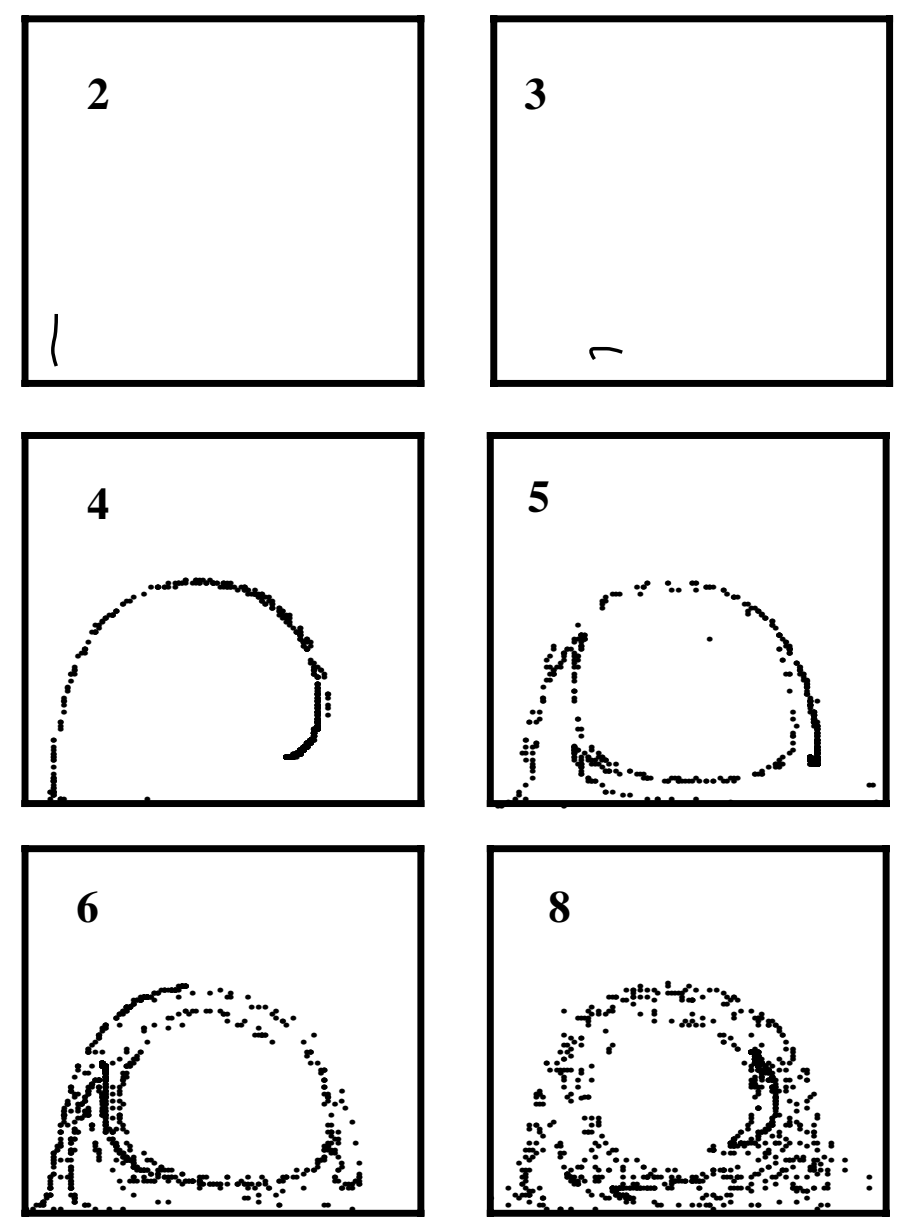

Figure 3. 


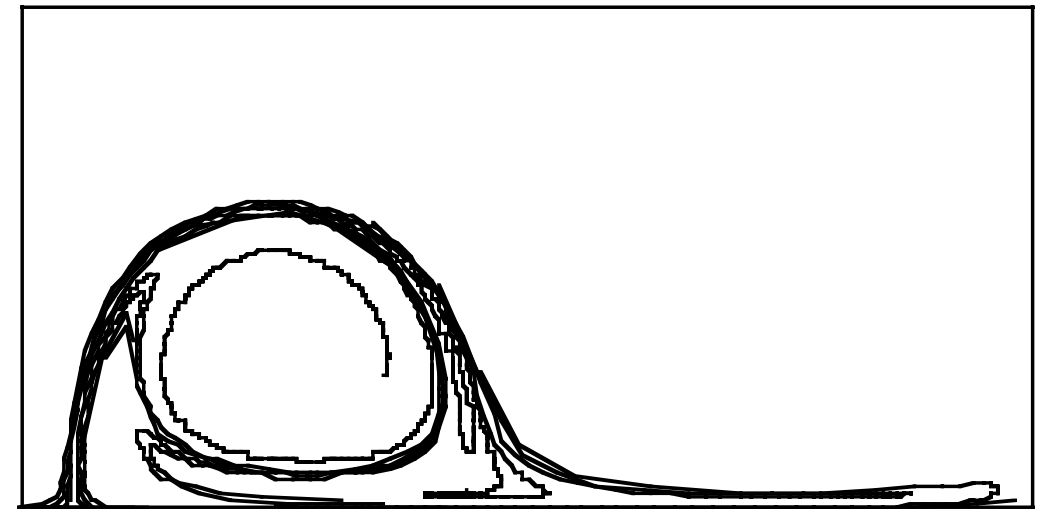

Figure $4 \ldots \ldots \ldots$. A segment of the unstable manifold, $\varepsilon=.15$.
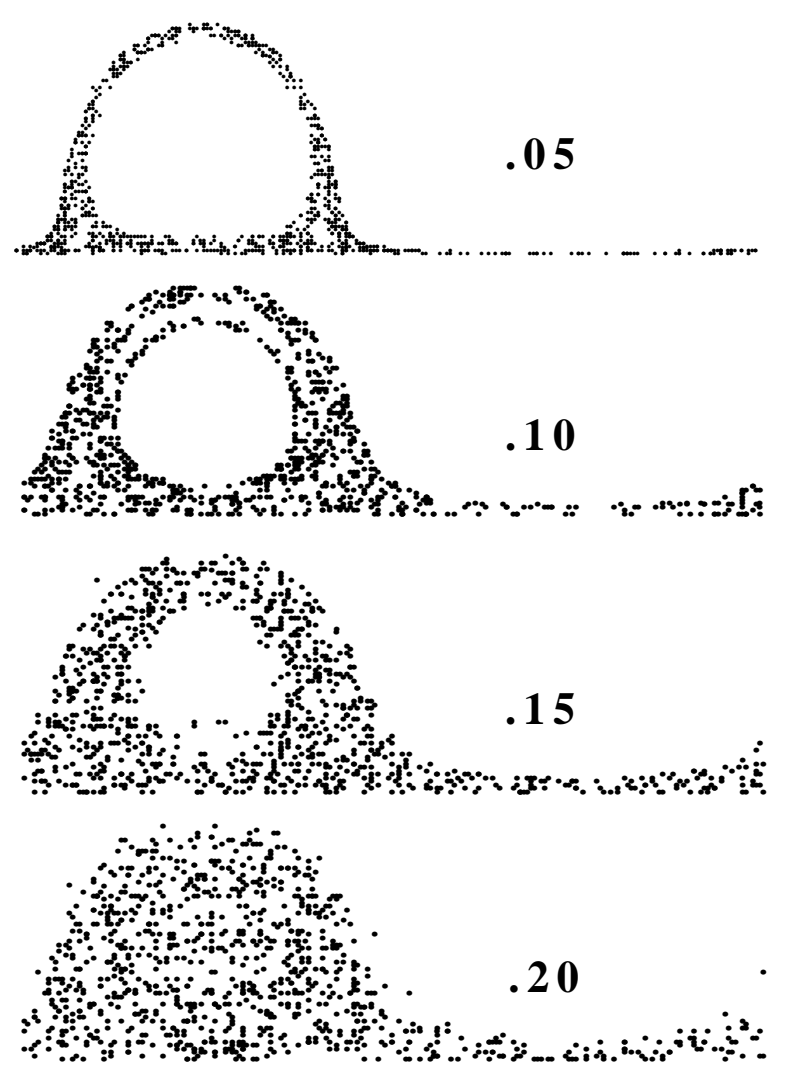

Figure 5............ Stochastic band vs. $\varepsilon$ for (top to bottom) $\varepsilon=.05, .10, .15, .20$ 


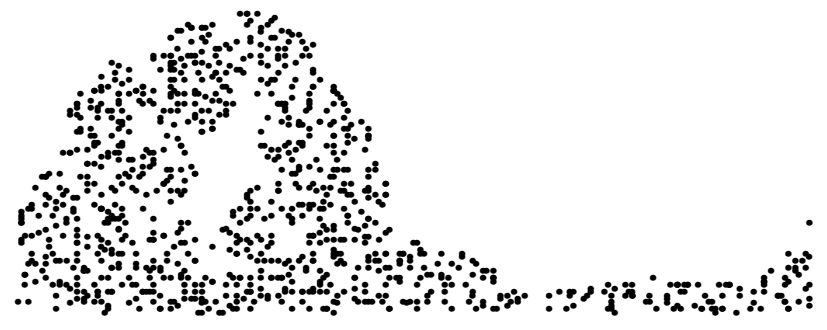

Figure 6.............Poincare' orbits for $\varepsilon=.05$, but with short wave perturbation having $\mathrm{k}_{2}=\mathrm{l}_{2}=4$.

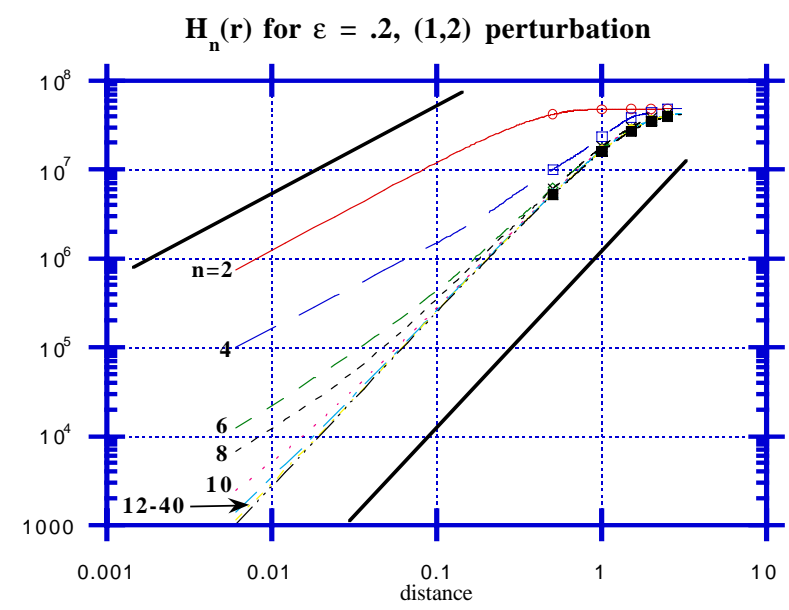

Figure 7............ Two particle correlation function for $(1,2)$ perturbation with $\varepsilon=.2$. The initial condition is a square of side .01 centered on $(\pi / 2,25)$. The bold lines have slopes 1 and 2 . 


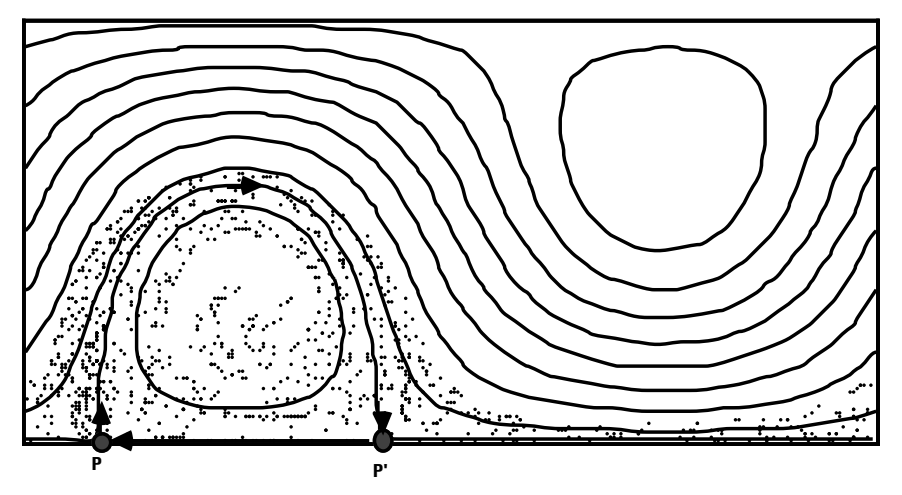

Figure 8.......... Tracer cloud at Poincare iterate $n=7$ for the case shown in Figure 7.

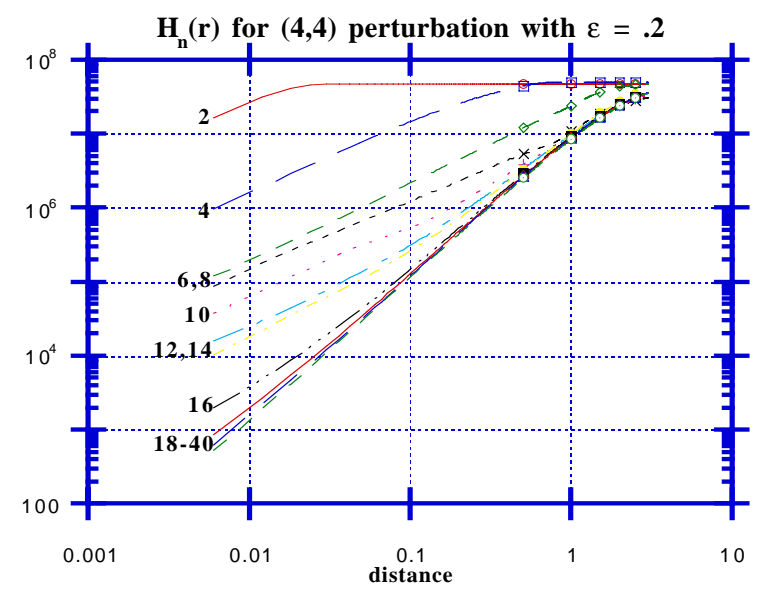

Figure 9............ As in Figure 7, but for $(4,4)$ perturbation with $\varepsilon=.2$. 


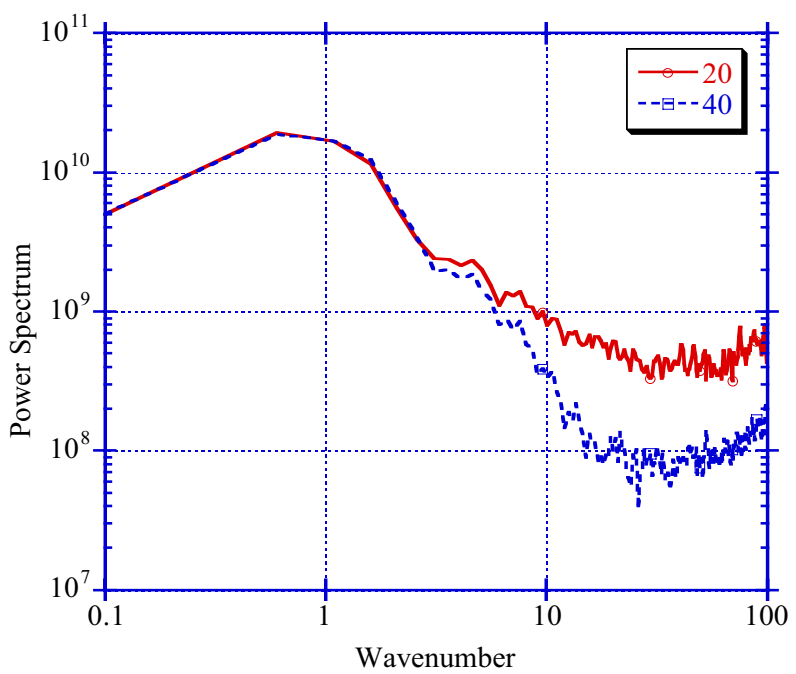

Figure 10........... Concentration power spectrum of tracer cloud at $\mathrm{n}=20$, and $\mathrm{n}=40$ for a $(1,2)$ perturbation with $\varepsilon=.25$ The initial condition is a cloud of 40000 points distributed uniformly over a square of side .01 centered on $(\pi / 2,1$.
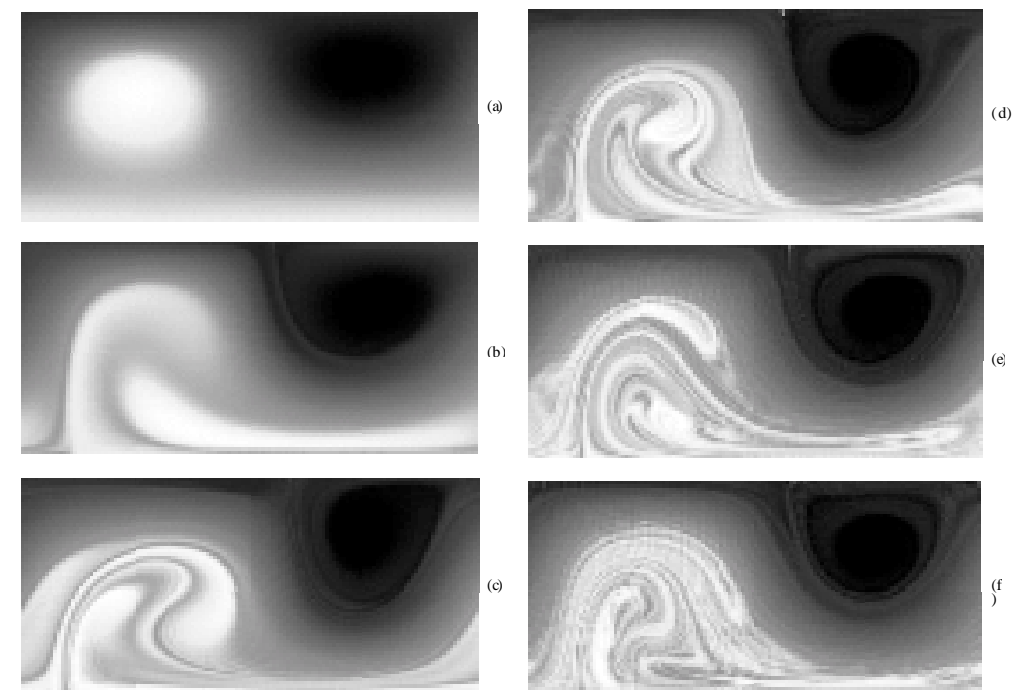

Figure 11 .Potential vorticity for case [1x2] at (a) $\mathrm{t}=0, \quad$ (b) $\mathrm{t}=7.8, \quad$ (c) $\mathrm{t}=15.6, \quad(\mathrm{~d})$ $\mathrm{t}=20.8$, (e) $\mathrm{t}=26.0$ (f) $\mathrm{t}=31.2$ 
(a)
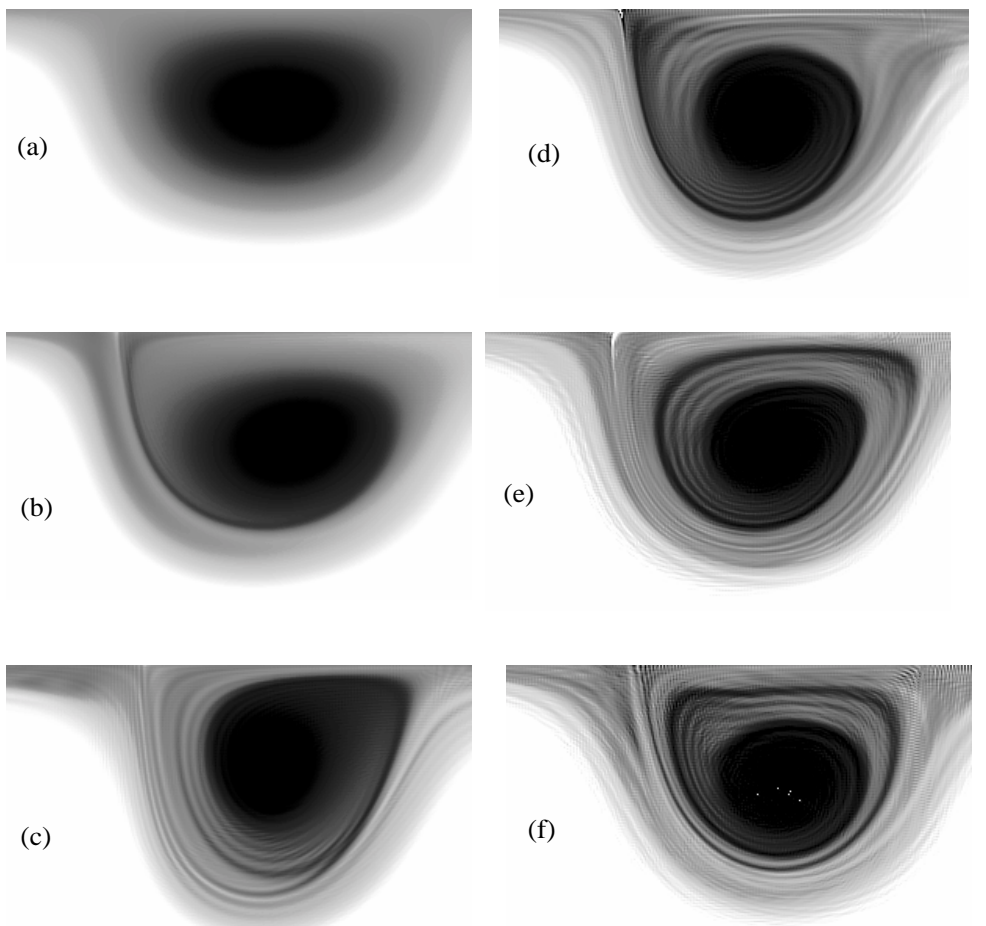

Figure 12.......... As in Figure 11, but remapped to emphasize the upper right vortex.
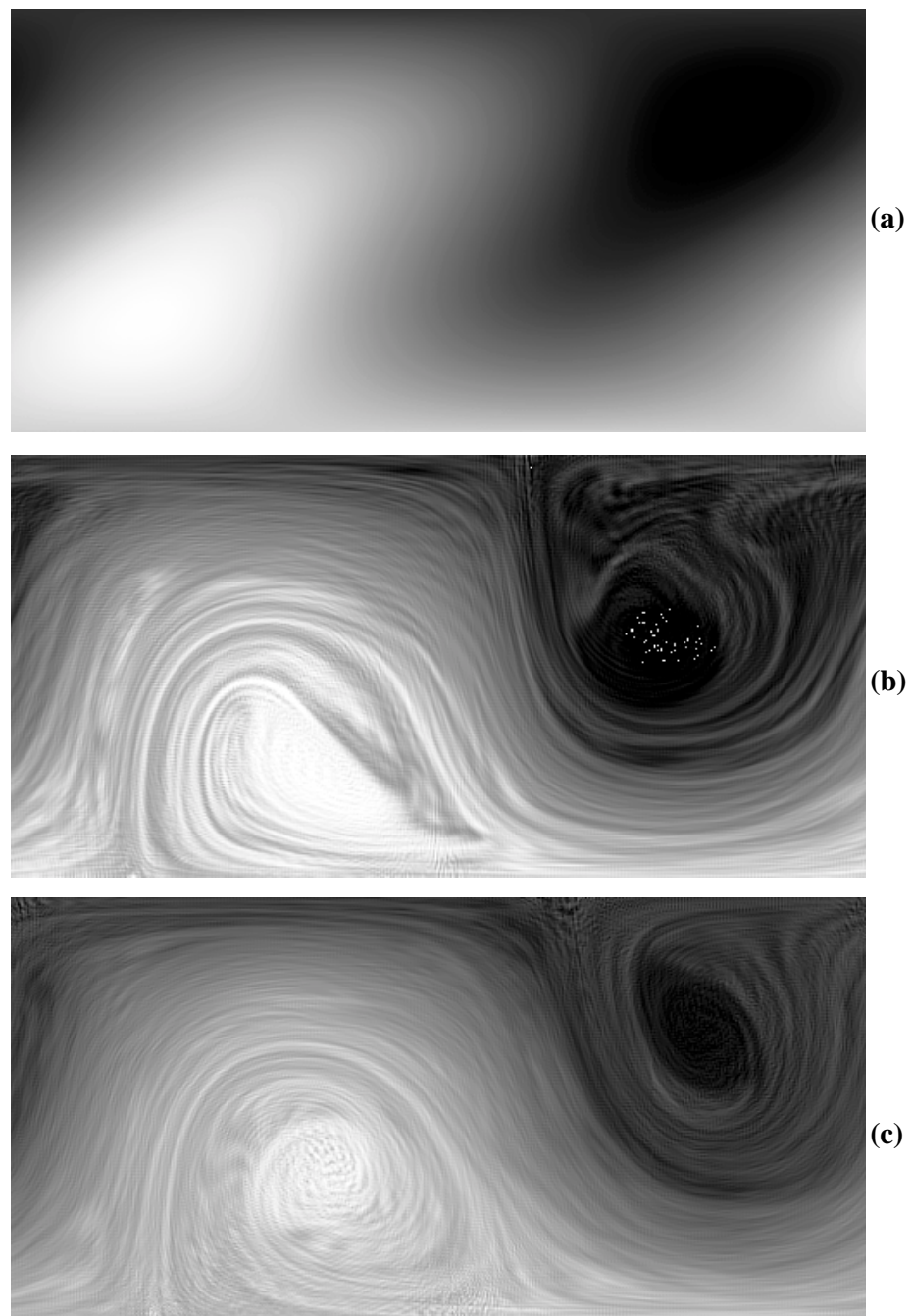

Figure $13 \ldots \ldots$.... Potential vorticity for case $[1 \times 2 s]$ at (a) $\mathrm{t}=0$, (b) $\mathrm{t}=39.0$ (c) $\mathrm{t}=63$ 

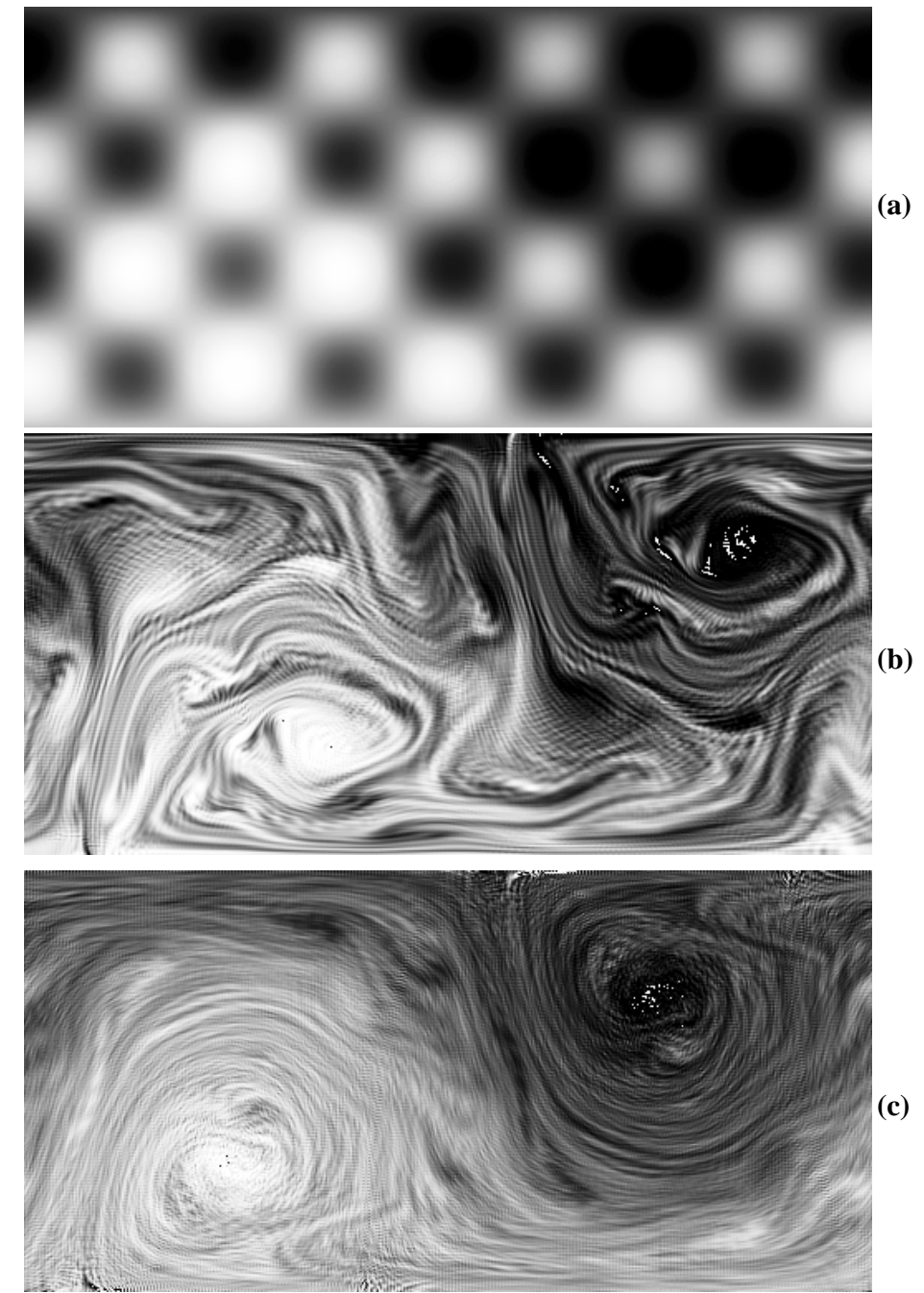

Figure 14

..Potential vorticity for case $[4 \times 4]$ at (a) $\mathrm{t}=0$, (b) $\mathrm{t}=10.4$, (c) $\mathrm{t}=31.2$

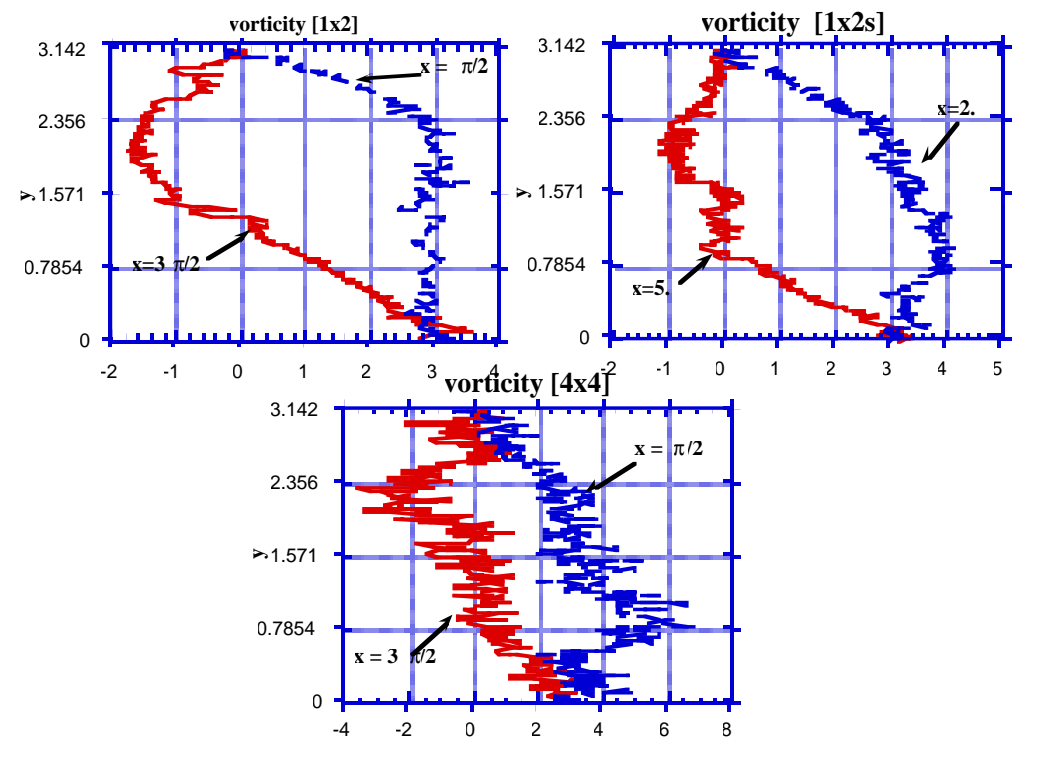

Figure 15 Vorticity cross sections along lines $\mathrm{x}=$ constant for cases [1x2], [1x2s] and [4x4], taken at the final times shown in Figs. 11-14. 

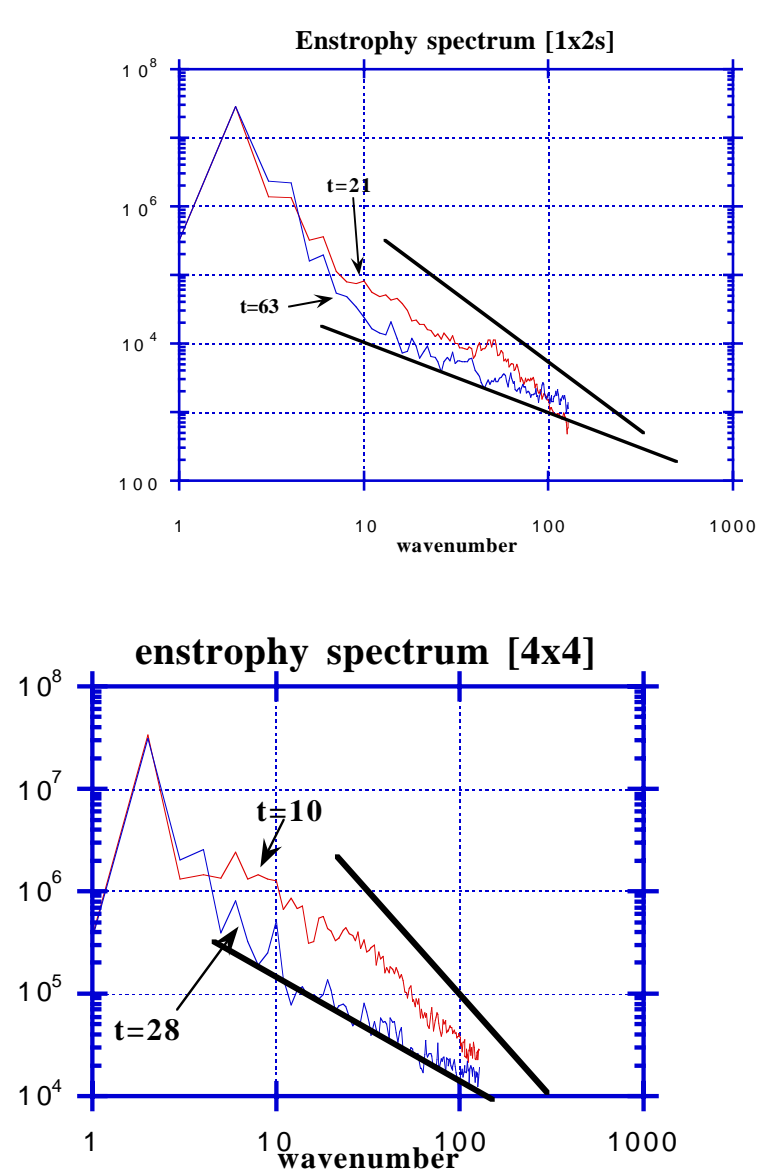

Figure 16......... Enstrophy spectra for (a) case [1x2s] and (b) case [4x4]. The bold lines have slopes 1 and 2 .
$\mathrm{E}(\mathrm{t})$ for $\mathrm{A}=1,4 \times 4$ wave perturbation,eps $=.2$

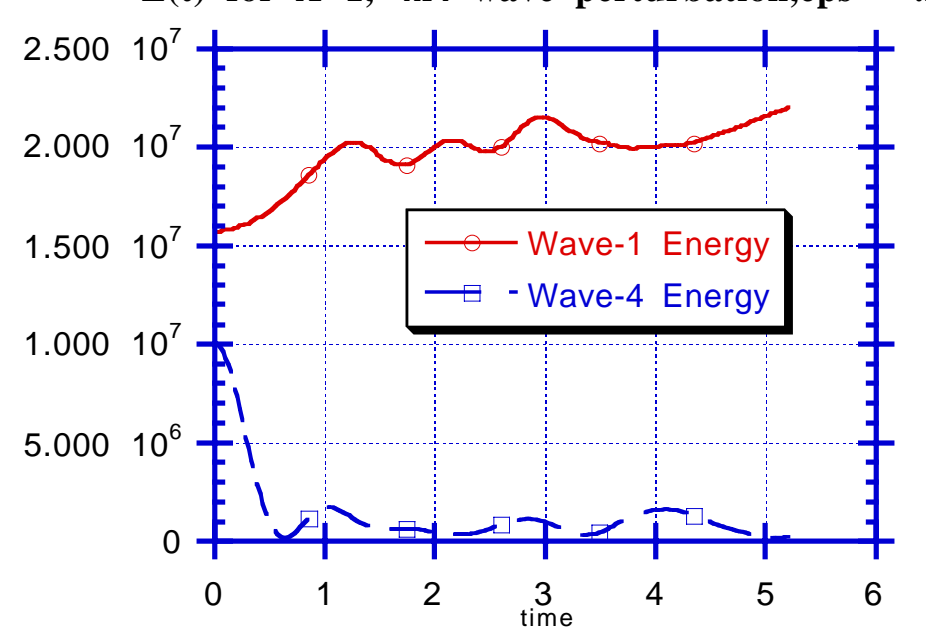

Figure $17 \ldots \ldots \ldots$....... energy $E_{n}(t)$ in zonal mode $n$ for $A=1$ subject to a $(4,4)$ perturbation with $\varepsilon=.2$. 


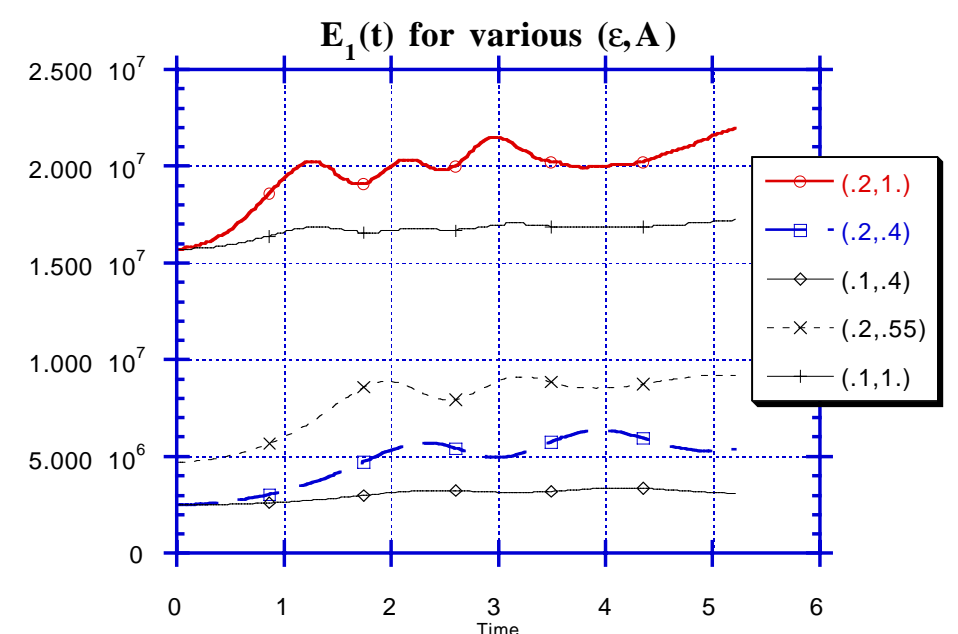

Figure 18.......... Energy $\mathrm{E}_{1}(\mathrm{t})$ in wave 1 for $(4,4)$ initial perturbation with various combinations ( $\varepsilon, \mathrm{A})$. 